\title{
Schwere Bewusstseinsstörungen in der neurologisch-neurochirurgischen Frührehabilitation
}

Neurol Rehabil 2016; 22(3): 192-208

(C) Hippocampus Verlag 2016

DOI 10.14624/NR150816.003

\author{
A. Bender ${ }^{1,2}$
}

\section{Zusammenfassung}

Schwere quantitative Bewusstseinsstörungen in Folge einer akuten Hirnschädigung treten klinisch entweder als Koma, Syndrom der reaktionslosen Wachheit (SRW, früher: apallisches Syndrom) oder Syndrom des minimalen Bewusstseins (SMB, engl.: Minimally Conscious State, MCS) in Erscheinung. Sie betreffen einen relevanten Anteil der Patienten der neurologisch-neurochirurgischen Frührehabilitation (NNFR) in Deutschland und stellen besondere Herausforderungen an die behandelnden Rehabilitationsteams dar. Diese reichen von der adäquaten Diagnose des Bewusstseinszustandes mittels standardisierter Verfahren (z. B. Coma Recovery Scale-Revised, CRS-R) über das medizinische Management der gehäuft vorkommenden Komplikationen, Untersuchungsmethoden zur Prognostizierung, interdisziplinäre therapeutische Maßnahmen bis hin zu Fragen der Angehörigenbetreuung und ggf. Entscheidungen am Lebensende. Zusätzlich bietet das Feld der moderne Komaforschung, z.B. mittels funktioneller Magnetresonanztomographie (fMRT) oder der elektrophysiologischen Analyse ereigniskorrelierter Potenziale vielfältige neue Erkenntnisse, die einerseits Diagnostik und Prognostizierung bereichern, andererseits aber auch zu einer zunehmenden Verunsicherung der Angehörigen führen könnten.

Die folgende Arbeit soll einen Überblick über diese verschiedenen klinisch relevanten Aspekte der Betreuung von Patienten mit schweren Bewusstseinsstörungen in der NNFR vermitteln.

Schlüsselwörter: Koma, Syndrom reaktionsloser Wachheit, Syndrom des Minimalbewusstseins, Prognose, Komaforschung

\section{Einleitung}

Patienten im Koma, Syndrom der reaktionslosen Wachheit (SRW, früher: apallisches Syndrom) oder Syndrom des minimalen Bewusstseins (SMB; syn.: Minimally Conscious State, MCS) stellen einen relevanten Teil des Patientenspektrums der neurologisch-neurochirurgischen Frührehabilitation (NNFR) in Deutschland dar. Auch wenn klare epidemiologische Daten zu Prävalenz und Inzidenz von schweren quantitativen Bewusstseinsstörungen fehlen, deuten aktuelle Ergebnisse aus Deutschland darauf hin, dass sie in der NNFR relativ häufig sind [78]. In dieser multizentrischen Studie betrug der durchschnittliche Wert auf der Glasgow Coma Skala (GCS) aller 754 eingeschlossenen Patienten bei Aufnahme in die NNFR 11,7 $\pm 3,6$ Punkte und lag somit deutlich im Bereich relevanter quantitativer Bewusstseinsstörungen. Wie viele Patienten tatsächlich die Definitionen von Koma, SRW oder MCS erfüllten, kann der Publikation leider nicht entnommen werden. Allerdings ist es bemerkenswert, dass selbst bei Entlassung noch 7,6\% der Patienten in der Glasgow Outcome Skala (GOS) einen Wert von 2 hatten und somit auch nach der NNFR noch einer dieser drei Kategorien von Bewusstseinsstörungen zuzuordnen waren. In unserem eigenen Patientengut erfüllten $76 \%$ der Patienten mit hypoxisch-ischämischer Enzephalopathie (HIE), 42\% der Patienten mit Schädel-Hirn-Trauma (SHT) und 25\% der Patienten mit einer Subarachnoidalblutung (SAB) bei Aufnahme die diagnostischen Kriterien von Koma, SRW oder MCS [40, 49, 50].

Die Behandlung von Patienten mit schweren Bewusstseinsstörungen ist somit relevant häufig in der Frührehabilitation. Der folgende Beitrag befasst sich mit den besonderen Aspekten von Diagnose, Behandlung, Prognostizierung, Langzeit-Behandlungsergebnis sowie medizin-ethischen Fragen bei dieser Patientengruppe im Rahmen der NNFR.

\section{Das Spektrum von Bewusstseinsstörungen}

Das Spektrum von schweren quantitativen Bewusstseinsstörungen reicht vom tiefem Koma über das SRW bis hin zum SMB/MCS (Abb. 1). Koma wurde ursprünglich definiert als die Unfähigkeit, Aufforderungen zu befolgen, zu sprechen oder die Augen zu öffnen [41]. Im Gegensatz zum Koma öffnen Patienten im SRW immer wieder die Augen, interagieren aber - zumindest bei rein klinischer Untersuchung - nicht reproduzierbar bewusst 


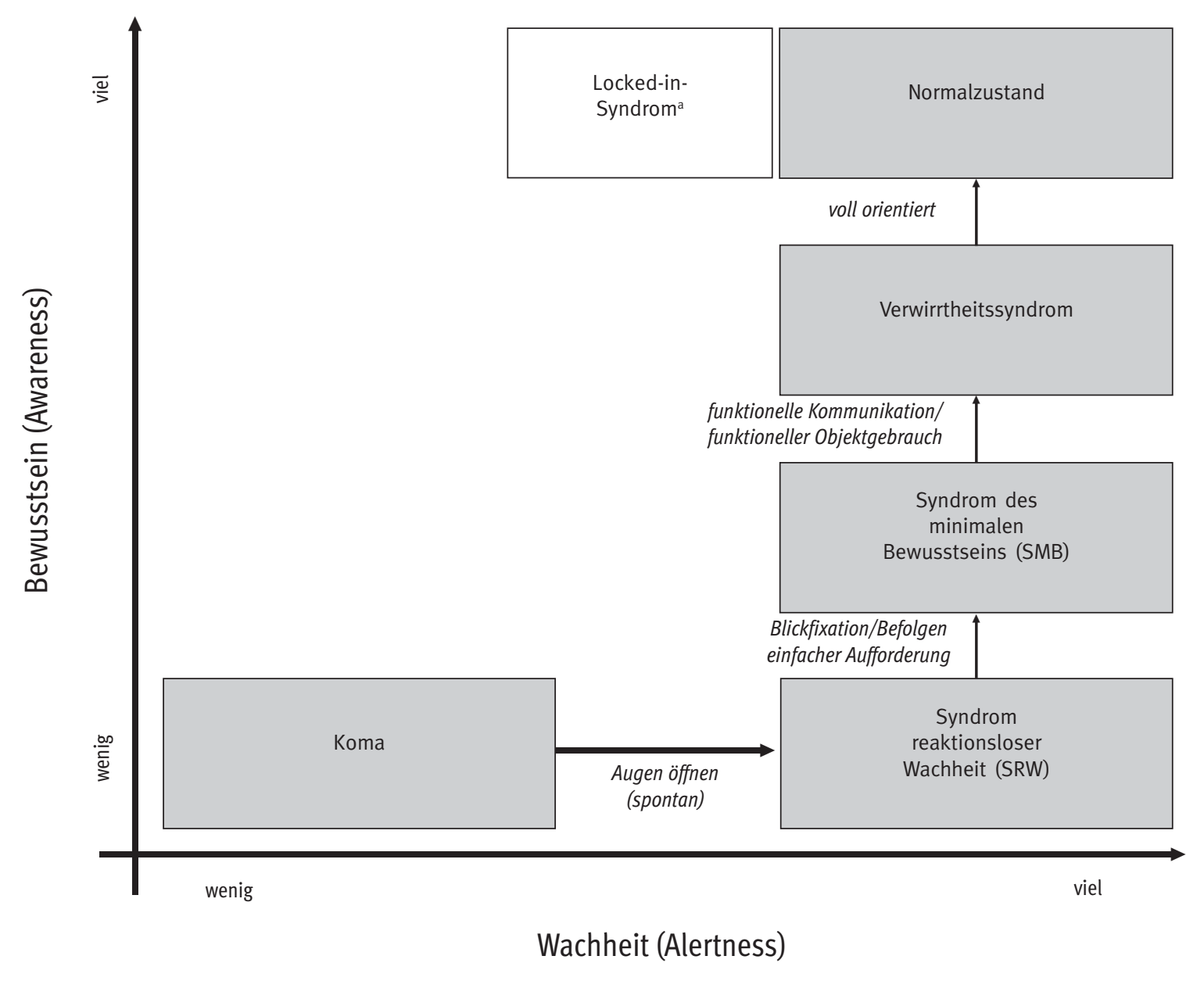

Abb.1: Zuordnung von verschiedenen Kategorien von Bewusstseinsstörungen zu den zwei Achsen Wachheit (Alertness) und Bewusstsein (Awareness). Der wesentliche Unterschied zwischen Koma und Syndrom der reaktionslosen Wachheit (SRW) sind bei letzterem die Phasen von Wachheit, charakterisiert durch geöffnete Augen. Eine basale Fähigkeit zur Kontaktaufnahme charakterisiert das Syndrom des minimalen Bewusstseins (SMB/MCS), funktioneller Objektgebrauch bzw. funktionelle Kommunikation dessen Überwinden. aPatienten im Locked-in-Syndrom (LIS) sind sowohl wach als auch kontaktfähig bei Bewusstsein. Auch wenn das LIS somit nicht zu den Bewusstseinsstörungen gehört, so ist es hier aufgeführt, um den Unterschied zu SRW und SMB zu verdeutlichen

mit ihrer Umgebung [57]. Vegetative oder motorische Reaktionen auf Schmerzen oder externe Stimuli werden jedoch häufig beobachtet. Patienten im SMB/MCS sind hingegen wiederholt kontaktfähig, wenn auch häufig auf eine sehr basale, allerdings nicht-reflexartige Weise [26]. Dies reicht vom Fixieren und Blickfolgebewegungen mit den Augen, einer gezielten Bewegung hin zum Schmerzreiz und situationsadäquatem Lächeln (sog. MCS/SMB Minus) bis hin zum bewussten Befolgen einfacher Aufforderungen (sog. MCS/SMB Plus).

Das Wiedererlangen einer funktionellen Kommunikationsfähigkeit oder der Fähigkeit zum funktionellen Objektgebrauch charakterisiert Patienten, die das SMB/ MCS überwunden haben. Häufig ist diese Phase durch ein hirnorganisches Psycho- bzw. Verwirrtheitssyndrom oder amnestisches Syndrom (engl.: Confusional State) gekennzeichnet.

Wichtig für das Verständnis und die Differenzierung dieser verschiedenen Bewusstseinsdiagnosen und ihrer
Differenzialdiagnosen ist die Tatsache, dass Wachheit (Alertness) und Bewusstsein (Awareness) unterschiedliche Aspekte eines klinischen Befundes darstellen (s. Abb. 1). Nur wenn sie in ausreichendem Maß gemeinsam gewährleistet sind, kann von einem erhaltenen Bewusstsein mit Kontaktfähigkeit ausgegangen werden.

Bereits an dieser Stelle sei darauf hingewiesen, dass das klassische Locked-in-Syndrom (LIS) bei Hirnstammläsionen keine Bewusstseinsstörung ist, da die Patienten kontaktfähig sind und nur durch den weitestgehenden Ausfall der Willkürmotorik als komatös verkannt werden können.

\section{Pathophysiologie}

Prinzipiell können schwere quantitative Bewusstseinsstörungen entweder durch strukturelle Hirnläsionen hervorgerufen werden oder aber nicht-strukturell durch schwere Beeinträchtigung der neuronalen Netzwerk- 


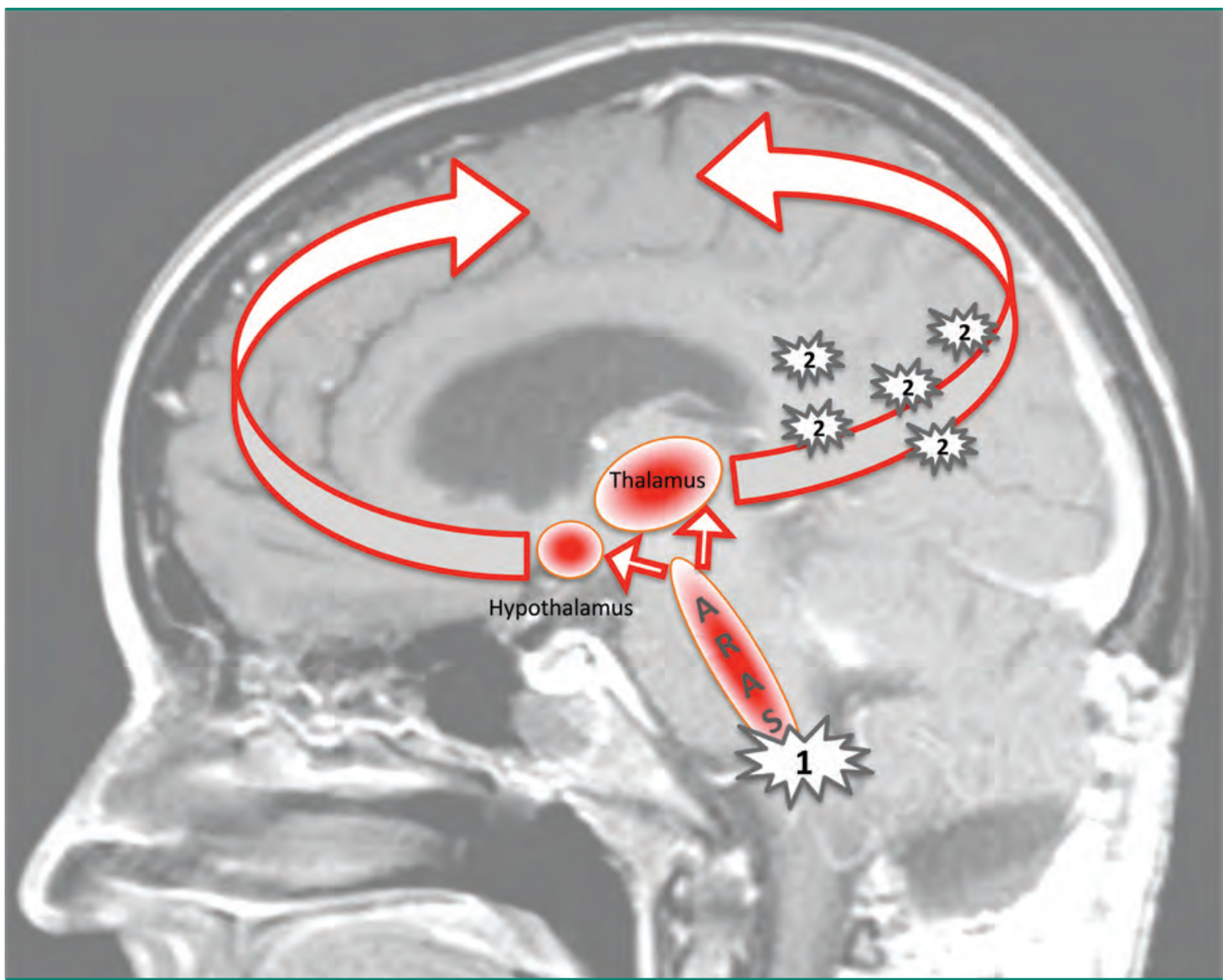

Abb. 2: Schematische Darstellung des Netzwerks, das für Wachheit (Alertness) und Bewusstsein notwendig ist. Das aufsteigende retikuläre Aktivierungssystem (ascending reticular activating system, ARAS; in rot markiert) entspringt in cholinergen Neuronen der Formatio reticularis im Hirnstamm und projiziert dann via Thalamus und Hypothalamus/basales Vorderhirn diffus in denKortex [109]. Sowohl strategische Einzelläsionen im Bereich des ARAS (z. B. mit 1 markiert bei Hirnstamminfarkt) wie auch diffuse Läsionen im Bereich der weiteren Projektionen (z. B. mit 2 markiert bei SHT mit diffuser axonaler Schädigung) können zu schweren Bewusstseinsschädigungen führen

funktionen im Rahmen von z.B. metabolischen Störungen oder medikamentös-toxisch [108, 110].

Bei strukturellen Läsionen als Koma-Ursache unterscheidet man strategische Einzelläsionen in Wachheits-relevanten Hirnstrukturen (z.B. Bilaterale Thalamusläsionen nach Basilariskopf-Embolie) oder aber ausgedehnte diffuse Hirnschädigungen, z. B. im Rahmen einer diffusen axonalen Schädigung nach SHT. Wichtiger Taktgeber für den aktiven Normalzustand eines bewussten Wachseins ist das aufsteigende retikuläre Aktivierungssystem (ascending reticular activating system, ARAS), das von der Formatio reticularis des Hirnstamms in einem vorderen und einem hinteren cholinergen Projektionssystem über den Thalamus oder den Hypothylamus den Cortex aktiviert [103] (Abb. 2). Strukturelle Läsionen im Bereich des ARAS können zu einer Unterbrechung der für die Wachheit notwendigen Bahnen und so zum klinischen Bild des Komas mit geschlossenen Augen führen.

\section{Klinische Diagnose des Bewusstseinszustandes}

Die klassische Skala zur Beurteilung des Bewusstseinsgrades ist die GCS [94]. Sie ist jedoch nur eingeschränkt verwertbar bei intubierten bzw. tracheotomierten Patienten (fehlende Möglichkeit zur verbalen Reaktion) und berücksichtigt nur unzureichend das Ausmaß einer möglichen Störung der Hirnstamm- und Hirnnervenfunktionen.

Aus diesem Grund wurde die FOUR-Skala (Full Outline of UnResponsiveness) entwickelt, die auch nonverbales Antwortverhalten sowie Hirnstammfunktionen berücksichtigt [107, 109]. Im Vergleich zur GCS- können mittels FOUR-Einstufung auf Grund der Möglichkeit zum nonverbalen Befolgen von Aufforderungen sowie zum basalen Kontaktfähigkeitsnachweis durch Blickfolgebewegungen mehr Patienten im SMB/MCS erkannt werden [11]. Weder GCS noch FOUR erlauben jedoch eine gezielte Differenzierung zwischen den verschiede- 
Tab. 1: Vergleich von Coma Recovery Scale-Revised (CRS-R) und Koma Remissions Skala (KRS)

\begin{tabular}{|c|c|c|}
\hline Kriterien & CRS-R & KRS \\
\hline Auditive Funktionen & $\begin{array}{l}\text { Bewegung konstant auf Aufforderung (4) }{ }^{\text {SMB }} \\
\text { Reproduziert Bewegung auf Aufforderung (3) SMB } \\
\text { Orientierungsreaktion auf Geräusche (2) } \\
\text { Schreckreaktion auf Geräusche (1) } \\
\text { Keine (0) }\end{array}$ & $\begin{array}{l}\text { Erkennt z.B. vertraute Stimme (3) } \\
\text { Augenöffnen/Kopfwenden/evtl. Lächeln (2) } \\
\text { Schreckreaktion (1) } \\
\text { Keine (0) }\end{array}$ \\
\hline Visuelle Funktionen & $\begin{array}{l}\text { Objekterkennung (5) SMB } \\
\text { Objektlokalisation (4) SMB } \\
\text { Blickfolge (3) SMB } \\
\text { Fixieren (2) } \\
\text { Visuelle Schreckreaktion (1) } \\
\text { Keine (0) }\end{array}$ & $\begin{array}{l}\text { Erkennen von Bildern/Personen/Gegenständen (4) } \\
\text { Verfolgt gezielt mit dem Blick (3) } \\
\text { Fixiert (2) } \\
\text { Gelegentliches, zufälliges Anschauen (1) } \\
\text { Keine (0) }\end{array}$ \\
\hline Motorische Funktionen & $\begin{array}{l}\text { Funktioneller Objektgebrauch (6) eMcS } \\
\text { Automatisierte motorische Reaktion (5) SMB } \\
\text { Objektmanipulation (4) SMB } \\
\text { Bewegung zum Schmerzreiz (3) SMB } \\
\text { Bewegung weg vom Schmerzreiz (2) } \\
\text { Pathologisches Muster (1) } \\
\text { Keine (0) }\end{array}$ & $\begin{array}{l}\text { Spontanes Greifen (6) } \\
\text { Gezielte Schmerzabwehr (5) } \\
\text { Körper-Haltereaktion erkennbar (4) } \\
\text { Ungezielte Schmerzabwehr (3) } \\
\text { Beugesynergismen (2) } \\
\text { Strecksynergismen (1) } \\
\text { Keine (0) }\end{array}$ \\
\hline $\begin{array}{l}\text { Oromotorische/Verbale } \\
\text { Funktionen } \\
\text { Sprechmotorische Antwort }\end{array}$ & $\begin{array}{l}\text { Verständliche Verbalisation (3) Sмв } \\
\text { Vokalisation/orale Bewegung (2) } \\
\text { Orale Reflexe (1) } \\
\text { Keine (0) }\end{array}$ & $\begin{array}{l}\text { Mind. } 1 \text { verständliches Wort (3) } \\
\text { Unverständliche Laute (2) } \\
\text { Stöhnen/Schreien/Husten (1) } \\
\text { Keine Phonation/Artikulation hörbar (0) }\end{array}$ \\
\hline Kommunikation & $\begin{array}{l}\text { Funktionell korrekt (2) emcs } \\
\text { Nicht Funktionell (1) SMB } \\
\text { Keine }(0)\end{array}$ & \\
\hline $\begin{array}{l}\text { Arousal/Erweckbarkeit/ } \\
\text { Aufmerksamkeit }\end{array}$ & $\begin{array}{l}\text { Aufmerksamkeit (3) } \\
\text { Augenöffnen spontan (2) } \\
\text { Augenöffnen mit Stimulation (1) } \\
\text { Nicht erweckbar (0) }\end{array}$ & $\begin{array}{l}\text { Aufmerksamkeit > } 1 \text { Minute (5) } \\
\text { Verweilen am Reiz > } 5 \text { Sek. (4) } \\
\text { Hinwendung zum Reiz (3) } \\
\text { Augenöffnen spontan (2) } \\
\text { Augenöffnen auf Schmerzreiz (1) } \\
\text { Nicht erweckbar (0) }\end{array}$ \\
\hline Reaktion auf taktile Reize & & $\begin{array}{l}\text { Erkennt durch Betasten/Fühlen ( } 3 \text { ) } \\
\text { Tastet spontan aber ohne Verständnis (2) } \\
\text { Vegetative Reaktion auf passive Berührung (1) } \\
\text { Keine (0) }\end{array}$ \\
\hline Min./Max. Summenwerte & $0 / 23$ & $0 / 24$ \\
\hline
\end{tabular}

nen Abstufungen von quantitativen Bewusstseinsstörungen, insbesondere zwischen SRW und SMB/MCS. In einer prospektiven Untersuchung an 60 Patienten mit schweren Bewusstseinsstörungen wurden nach reiner GCS-Einstufung $n=29$ Patienten als SRW kategorisiert [83]. Mittels FOUR wurden 4 dieser 29 als SMB/MCS eingestuft, aber bei Verwendung der Coma Recovery Scale in ihrer revidierten Fassung (CRS-R) zeigte sich, dass im Vergleich zur FOUR 7 weitere Patienten im SMB/MCS waren $[46,83]$.

Die CRS-R beurteilt das Reaktionsvermögen anhand von sechs verschiedenen Subskalen, die am Ende einen Summenscore von o (völliges Fehlen von Reaktionen) bis hin zu 23 Punkten (adäquat kontaktfähig mit funktioneller Kommunikation) ergeben. Zusätzlich ist das Erreichen eines SMB/MCS bzw. dessen Überwinden anhand verschiedener Items klar operationalisiert (Tab. 1). Die CRS-R erlaubt somit eine vergleichbare Beschreibung und diagnostische Zuordnung des Bewusstseinszustandes und ist mittlerweile der Goldstandard in der internationalen Komaforschung, auch wenn sie ebenfalls Nachteile aufweist, z.B. die mögliche Unterschätzung des Bewusstseinszustandes bei Patienten mit schwerer Aphasie und mit fehlenden motorischen Antwortmöglichkeiten [28, 60, 82]. Ein Summenscore von 10 Punkten oder mehr auf der CRS-R identifiziert $78 \%$ der SMB/MCS Patienten bzw. derjenigen Fälle, die das SMB/MCS überwunden haben (Sensitivität). Mit diesem Cut-off-Wert beträgt die Spezifität $100 \%$, d.h. kein SRW-Patient wird zu Unrecht als SMB/MCS klassifiziert [9]. Die CRS-R liegt seit mehreren Jahren auch in einer deutschsprachigen Version vor, zu der es auch Schulungsvideos gibt [65].

In deutschsprachigen Ländern wird in der NNFR hingegen in den meisten Fällen die Koma-RemissionsSkala (KRS) benutzt [72]. KRS und CRS-R ähneln sich in vielen Items stark, sind jedoch nicht deckungsgleich. 


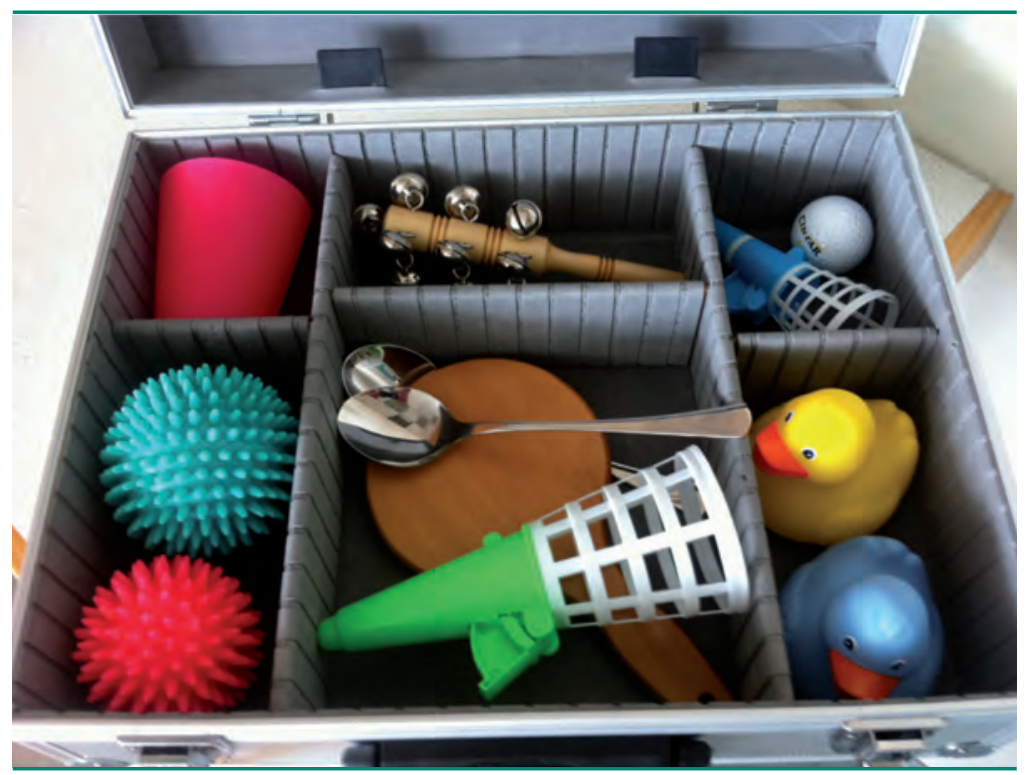

Abb. 3: Beispiel für in Burgauer Klinik verwendete Materialien zur Beurteilung des Bewusstseinszustandes. Beachten Sie, dass die bloße Verwendung einer Pupillenleuchte und des Untersucherfingers nicht ausreicht, um eine differenzierte Bewusstseinsdiagnose zu stellen stand von Patienten adäquat beurteilen zu können. Diese Untersuchung kann sehr zeitaufwendig sein und erfordert es, den Patienten durch unterschiedliche Reize und Materialien die Möglichkeit zur Reaktion zu geben (Abb. 3). Bedacht werden sollte, dass der okzipitale Kortex aus ungeklärten Gründen zu den besonders vulnerablen Regionen bei einer HIE gehört. Die Folge ist, dass einige HIE-Patienten kortikal blind sind und dem im Rahmen der Kurzuntersuchung vorgehaltenen Finger deswegen nicht folgen können [3]. Aber auch bei Intaktheit der Sehbahn gibt es bessere Reize zum Auslösen einer Blickfolgebewegung als den vorgehaltenen Finger oder die Pupillenleuchte. Am meisten bewährt hat sich dabei das Verwenden eines Spiegels (Patient betrachtet Spiegelbild im langsam vor ihm bewegten Spiegel) oder alternativ das Vorhalten eines Smartphone-Bildschirms mit aktivierter Selfie-Kamera [17, 102]. Zu bedenken ist bei der klinischen Untersuchung, dass die Patienten häufig deutlich mehr Zeit zum Befolgen von Aufforderungen benötigen und dass ihre Leistungen oft nicht konstant im Tagesverlauf abrufbar sind [16, 88]. Eine einmalig negative klinische Untersuchung kann daher nicht als ausreichender Nachweis einer fehlenden Kontaktfähigkeit gewertet werden.

Insbesondere liegen für die KRS keine klar operationalisierten diagnostischen Kriterien für die Differenzierung zwischen SRW und SMB/MCS vor. Die KRS wird vielmehr zur intraindividuellen Verlaufsbeurteilung benutzt und kann hier auch sehr zielgerichtet Verbesserungen des Bewusstseinszustandes klar operationalisiert dokumentieren [72]. Dennoch wäre es aus Sicht des Autors wünschenswert, wenn auch in Deutschland vermehrt die international verwendete CRS-R Anwendung finden würde bzw. wenn auch für die KRS klare Kriterien für das Erreichen der verschiedenen Bewusstseinsdiagnosen definiert würden.

Es existieren noch weitere klinische Beurteilungssysteme und Skalen, die eine differenzierte Charakterisierung des Bewusstseinszustandes erlauben. Hier ist in erster Linie die SMART-Beurteilung (Sensory Modality Assessment and Rehabilitation Technique) zu erwähnen [30]. Sie erfordert jedoch ein hohes Maß an Schulung und hat in der klinischen Routineversorgung den großen Nachteil, dass ihre Durchführung sehr zeitaufwendig sein kann.

Die oben erwähnten standardisierten klinischen Bewertungssysteme sind zwingend erforderlich, um zur richtigen Bewusstseins-Diagnose zu kommen. Dies wird insbesondere dadurch verdeutlicht, dass die Rate an Fehldiagnosen erschreckend hoch ist und in manchen Studien mit bis $\mathrm{zu} \mathrm{43 \%}$ angegeben wird [3, 85, 101]. Schwierigkeiten bereiten insbesondere die Abgrenzung von SRW zu SMB/MCS aber auch die zwischen Koma/ SRW und Locked-in-Syndrom. Hier muss selbstkritisch angemerkt werden, dass die kurze klinisch-neurologische Untersuchung im Rahmen der täglichen Klinikvisite oft rein zeitlich nicht ausreicht, um den Bewusstseinszu-

\section{Ursachen und "Koma-Mimics»}

Es gibt keine genauen epidemiologischen Daten über die Häufigkeit der verschiedenen Ursachen für schwere Bewusstseinsstörungen bei Aufnahme in die NNFR. Vorläufige Ergebnisse einer prospektiven multizentrischen Registerstudie aus Deutschland, in die nur Patienten eingeschlossen werden, die bei Aufnahme in die NNFR entweder im Koma, SRW oder SMB sind, zeigen die HIE mit 45\% als häufigste Ursache, gefolgt von Schlaganfällen (inkl. intrazerebrale Blutungen und SAB) mit 31\% und SHT mit 24\% [33].

Die Gründe für nicht-traumatisch bedingte Bewusstseinsstörungen im Schockraum- bzw. Intensivstationssetting wurden in einer Metaanalyse mit mehr als 5.00o Patienten systematisch untersucht: Die häufigsten Ursachen waren Schlaganfälle (6-54\%), HIE (3-42\%), Intoxikationen (1-39\%), metabolische Veränderungen (1-31\%), epileptische Anfälle (2-13\%) und Infektionen (2-9\%) [39].

Eine nach unseren Erfahrungen in diesen Daten für die Situation in der NNFR im deutschsprachigen Raum unterrepräsentierte Ätiologie sind Bewusstseinsstörungen im Rahmen einer septischen Enzephalopathie, die oft parallel mit einer Critical-Illness-Polyneuropathie bzw. -Myopathie (CIP/CIM) auftreten [69, 99]. Bedingt durch die Struktur der NNFR im Rahmen des Phasenmodells der Bundesarbeitsgemeinschaft für Rehabilitation in Deutschland scheint die CIP/CIM zu einer der Haupt-Aufnahmediagnosen zu werden. War die CIP bei einer Erhebung 2002 noch gar nicht als eigenständige Hauptdiagnose aufgeführt, so lag ihr Anteil 2014 bereits bei $17 \%$, und sie repräsentierte damit die zweithäufigste 
Aufnahmediagnose in der NNFR [38, 77, 78]. Dies mag insbesondere auch daran liegen, dass es für diese primär nicht-neurologische Patientengruppe in den anderen Fachgebieten keine zur Neurologie vergleichbaren Rehabilitationsstrukturen gibt.

In den meisten Fällen wird die Ätiologie der Bewusstseinsstörung bei Aufnahme in die NNFR durch die vorangegangene Diagnostik in der Akutaufnahmesituation bereits geklärt sein. Dennoch kann es sinnvoll sein, bei unerwartet lang anhaltenden oder in der NNFR neu aufgetretenen Bewusstseinsminderungen auch nach selteneren und nicht zwingend primär neurologischen Ursachen zu suchen (Tab. 2).

Wichtig ist die Differenzierung zwischen »echten« Bewusstseinsstörungen und einer nur augenscheinlichen motorischen Reaktionslosigkeit der Patienten (»Koma-Mimics«). Die diagnostisch größte Herausforderung scheint hierbei die Abgrenzung vom LIS zu sein [3, 15]. Bei Patienten mit bekannter Hirnstamm-Pathologie (z. B. Basilaristhrombose, Hirnstammblutung oder Hirnstammenzephalitis) und fehlender Kontaktfähigkeit muss daher viel Zeit auf die klinisch-neurologische Untersuchung verwendet werden, um erhaltene (vertikale) Augenbewegungen auf Aufforderung nicht zu übersehen. Gegebenenfalls muss zusätzlich ein EEG durchgeführt werden, um eine Inkongruenz zwischen gut erhaltener Reaktivität und Grundrhythmus sowie dem vermeintlich komatösen klinischen Bild aufzudecken.

Auch hypokinetisch-rigide extrapyramidale Störungen können den Eindruck einer schweren Bewusstseinsstörung vermitteln und sollten bei entsprechender Anamnese ausgeschlossen werden. Ein »Klassiker» ist das präoperative Absetzen bzw. das ausbleibende Wiederansetzen dopaminerger Medikamente bei Parkinson-Patienten. Auch die Verabreichung nicht-sondengängiger Levodopa-Präparate (z.B. Madopar ${ }^{\circledR}$ anstelle Madopar LT $^{\circledR}$ ) kann zu einer hypokinetischen Krise führen, die als Koma oder SRW verkannt wird.

Bei psychiatrischer Komorbidität oder ausgeprägten frontalen Läsionen kann auch ein akinetischer Mutismus mit einem SRW verwechselt werden.

Schließlich kann es in seltenen Fällen auch zur Entwicklung einer medikamentös-getriggerten myasthenen Krise kommen, selbst bei Patienten ohne vorbekannte derartige motorische Endplattenerkrankung. Häufigste auslösende Substanz ist dabei D-Penicillamine [5].

\section{Gerätegestützte Zusatzdiagnostik}

Ziel der zusätzlichen gerätegestützten Diagnostik in der NNFR ist zum einen der Nachweis behandelbarer Ursachen oder von Gründen für eine ausbleibende Besserung und zum anderen das Sammeln von prognoserelevanten Informationen.

Bei den Standardverfahren spielen in der NNFR insbesondere die CT-/MRT-Diagnostik sowie EEG- und
Tab. 2: Ursachen für schwere Bewusstseinsstörungen jenseits der primären akuten Hirnschädigung

\begin{tabular}{|c|c|}
\hline Ursache & Bemerkung \\
\hline $\begin{array}{l}\text { Rezidiv Schlaganfall (inkl. } \\
\text { ICB) }\end{array}$ & $\begin{array}{l}\text { gehäuft bei Beginn/Unterbrechungen } \\
\text { der Antikoagulation }\end{array}$ \\
\hline Hydrozephalus & $\begin{array}{l}\text { insbesondere nach SAB oder } \\
\text { Raumforderung im Bereich der } \\
\text { Liquorabflusswege }\end{array}$ \\
\hline $\begin{array}{l}\text { Septische Enzephalo- } \\
\text { pathie }\end{array}$ & $\begin{array}{l}\text { oft Bewusstseinsminderung zeitlich vor } \\
\text { einem signifikanten Anstieg der Entzün- } \\
\text { dungszeichen }\end{array}$ \\
\hline medikamentös-toxisch & $\begin{array}{l}\text { insbesondere Neuroleptika, Antidepres- } \\
\text { siva (serotonerge und anticholinerge } \\
\text { Syndrome) }\end{array}$ \\
\hline endokrinologisch & $\begin{array}{l}\text { z.B. Hypothyreose durch fehlende Sub- } \\
\text { stitution oder auch Hypocortisolismus } \\
\text { durch traumatische Störung der Hypo- } \\
\text { thalamus-Hypophysen-Achse }\end{array}$ \\
\hline $\begin{array}{l}\text { nicht-konvulsiver Status } \\
\text { epilepticus }\end{array}$ & $\begin{array}{l}\text { nur durch EEG zu diagnostizieren; } \\
\text { mutmaßlich unterdiagnostiziert }\end{array}$ \\
\hline metabolisch & $\begin{array}{l}\text { renale oder hepatische Enzephalopa- } \\
\text { thie; Hyponatriämie; Hypo- und Hyper- } \\
\text { glykämie }\end{array}$ \\
\hline $\begin{array}{l}\text { Ventrikulitis bei bei } \\
\text { implantiertem Shunt- } \\
\text { System }\end{array}$ & $\begin{array}{l}\text { Zur Diagnose Liquorentnahme aus dem } \\
\text { Shunt-System; lumbaler Liquor kann } \\
\text { blande sein }\end{array}$ \\
\hline $\begin{array}{l}\text { Zentrale Pontine Myelino- } \\
\text { lyse (ZPM) }\end{array}$ & $\begin{array}{l}\text { Ausgelöst durch zu raschen Natrium- } \\
\text { ausgleich }\end{array}$ \\
\hline $\begin{array}{l}\text { Hypovitaminose, i.b. Vita- } \\
\text { min- } B_{1} \text {-Mangel }\end{array}$ & $\begin{array}{l}\text { Insbesondere bei schädlichem Gebrauch } \\
\text { von Alkohol oder massiven Glukoseinfu- } \\
\text { sionen ohne Thiamin-Zusatz }\end{array}$ \\
\hline
\end{tabular}

$\mathrm{ICB}=$ Intrazerebrale Blutung; $\mathrm{SAB}=$ Subarachnoidalblutung

SEP-Untersuchungen eine wichtige Rolle. Die Schnittbildverfahren sollen neue strukturelle Läsionen oder möglicherweise therapierelevante Hinweise auf Liquorzirkulationsstörungen als Ursache einer sekundären Verschlechterung oder eines stagnierenden Rehabilitationsverlaufes nachweisen. Sie können auch durch den Nachweis einer progredienten Hirnatrophie Informationen über das Ausmaß einer in den Standard-Schnittbildtechniken in der Frühphase nur schwer darstellbaren diffusen Hirnschädigung beisteuern [32, 34, 100]. Neben dem Ausmaß der kortikalen Atrophie scheinen insbesondere Volumenminderungen im Thalamus mit dem klinischen Bewusstseinszustand zu korrelieren [22]. Da quantitative volumetrische Verfahren noch keinen Eingang in die Routineversorgung gefunden haben, sind diese Informationen doch allenfalls qualitativ zu werten.

Die Domäne des EEG während der Frührehabilitation von Patienten mit schweren Bewusstseinsstörungen ist der Nachweis von epileptischer Aktivität im Sinne eines nicht-konvulsiven Status epilepticus (NKSE). Dieser wird vermutlich nach wie vor zu selten diagnostiziert [47, 54]. Der Nachweis eines NKSE sollte unbedingt eine konsequente antikonvulsive Therapie nach sich ziehen und nicht gleich automatisch als Nachweis einer infaust 
schweren Hirnschädigung mit entsprechendem therapeutischem Nihilismus interpretiert werden. Ansonsten droht die Gefahr einer »self fulfilling prophecy«, obwohl selbst $10 \%$ der HIE-Patienten mit einem NKSE bei entsprechender Behandlung eine gute Prognose haben können [25, 8o]. Selbstverständlich belegen diese Studien auch die Rolle eines Status epilepticus als negativem Prognosemarker, so dass wir an dieser Stelle lediglich vor der Gefahr eines therapeutischen Nihilismus warnen möchten. Möglicherweise ist der Nachweis einer fehlenden Hintergrund-Reagibilität im EEG jedoch ein noch stärkerer Prädiktor für ein schlechtes Outcome, zumindest nach HIE [81].

Die wenigsten Einrichtungen zur NNFR werden über die Möglichkeiten zur Durchführung innovativer und aufwendiger Verfahren zur Bewusstseinsdiagnostik, wie z.B. funktionelle Magnetresonanztomographie (fMRT) oder Positronenemissionstomographie (PET) verfügen. Auch wenn sich durch die Komaforschung der jüngsten Vergangenheit hier aussichtsreiche Verfahren zur Verbesserung der diagnostischen Treffsicherheit der Bewusstseinsdiagnose oder zur Prognosevorhersage abzeichnen, so sind diese momentan doch noch sehr weit von der klinischen Routineversorgung entfernt und daher nicht im Fokus dieser Arbeit (zur Übersicht: [6]). Kurz zusammengefasst konnte gezeigt werden, dass Ausmaß und Verteilungsmuster der Tracer-Bindung im FDG-PET bei Patienten im Koma, SRW oder SMB/MCS gut geeignet waren, um SMB/MCS-Patienten zu identifizieren, und mit der Prognose bezüglich Wiedererlangen des Bewusstseins im Einjahresverlauf korrelierte [92]. Zusätzlich besaßen $17 \%$ bis $32 \%$ der Patienten, die sich klinisch im SRW befanden und somit nicht kontaktfähig waren, im fMRT die Fähigkeit, ihre Hirnaktivität auf Aufforderung (Bewegungsvorstellung/räumliche Vorstellung) zu modulieren (gemessen durch Veränderungen des BOLD-Signals) bzw. im FDG-PET typische Aktivitätsmuster von SMB/MCS-Patienten [66, 92].

Auch die Struktur der NNFR in Deutschland mit oft dezentralen, isolierten Behandlungseinheiten »auf der grünen Wiese« unterstützt nur selten die für eine Weiterentwicklung der klinischen Versorgung notwendige enge Verzahnung zwischen rehabilitativer Patientenversorgung und wissenschaftlicher Forschung in diesen spezialisierten High-Tech-Bereichen.

Etwas anders stellt sich die Entwicklung von elektrophysiologischen Methoden dar, die auch in dezentralen Einrichtungen der NNFR bettseitig zur Verfügung gestellt werden können, ohne dass Patienten aufwendig $\mathrm{zu}$ Bildgebungszentren verlegt werden müssten. Mit relativ simplen und gut standardisierbaren Methoden für die Beurteilung ereigniskorrelierter Potenziale oder für die Untersuchung der Konnektivität zwischen verschiedenen Hirnarealen können durchaus auch in diesem Setting wertvolle prognostische Informationen gesammelt werden $[87,93]$. So weist z. B. die über die Ableitung der
N40o-Welle im EEG nachweisbare Fähigkeit zur semantischen Satzanalyse auf ein späteres Wiedererlangen des Bewusstseins hin [93]. Konnektivitäts- und Netzwerkanalysen auf Basis eines kurzen Ruhe-EEGs korrelieren außerdem mit der Wahrscheinlichkeit eines späteren Überwindens von SRW oder SMB/MCS [87].

\section{Behandlungsmöglichkeiten}

Die Basis der Therapie von Patienten mit schweren Bewusstseinsstörungen in der NNFR bildet das geschulte und empathische interdisziplinäre Behandlungsteam aus Rehabilitationsmedizinern, geschulten Fachpflegekräften und Therapeuten, insbesondere aus den Bereichen Physiotherapie, Ergotherapie, Sprach- und Schlucktherapie, Musiktherapie und Neuropsychologie (keine abschließende Aufzählung) [19, 27]. Der gemeinsame Ansatz ist das Ziel, durch gezielte Stimulation die Patienten wieder näher an ein Bewusstsein heranzuführen [98]. Generell ist die wissenschaftliche Evidenz in diesem Bereich für oder gegen eine bestimmte Therapieform oder -schule sehr gering, so dass sich oft klinikinterne Behandlungsgrundsätze auf dem Boden des eigenen langjährigen Erfahrungsschatzes gebildet haben [51]. Die Bedeutung eines funktionierenden interdisziplinären Teams mit einem vernetzten und umfassenden Therapiekonzept wird dann klar, wenn berücksichtigt wird, dass kein Einzeltherapiebaustein allein im Rahmen der 300 Therapieminuten pro Tag gemäß OPS 8-552 geeignet ist, bei diesen extrem schwer betroffenen Patienten zu einer signifikanten Verbesserung zu führen. Notwendig sind nach Überzeugung des Autors Therapiekonzepte, die die Patienten nach Möglichkeit über einen Großteil des Tages einbinden. Hierzu zählen Faktoren der Umweltgestaltung (»enriched environment«) genauso wie Lagerungsverfahren, gezielte Einzeltherapiebausteine und auch entsprechende pharmakologische Stimulationsversuche $[51,56]$. Häufig verwendete und aus Sicht des Autors auch bewährte therapeutische Konzepte für diese Patientengruppe sind Lagerungsverfahren (z.B. nach Bobath oder Lagerung in Neutralstellung), gespürte Interaktionstherapie zur Wahrnehmungsförderung nach Affolter ${ }^{\circledR}$, möglichst frühzeitige und häufige Vertikalisierung sowie die Stimulation des Facio-oralen Traktes [1, 2, 8, 36, 52, 75, 90].

Viele therapeutische Konzepte und randomisierte klinische Studien zur Outcome-Verbesserung von komatösen Patienten haben die ersten Stunden und Tage nach der akuten Hirnschädigung im Fokus, so z.B. die therapeutische Hypothermie bei HIE oder aber Interventionsstudien beim SHT [18, 91]. In der vorliegenden Arbeit sollen hingegen kurz diejenigen randomisierten und kontrollierten klinischen Interventionsstudien bzw. -verfahren referiert werden, die im frühen neurorehabilitativen Setting erfolgt sind (Tab. 3). Leider fehlen $\mathrm{zu}$ diesem Thema nach Kenntnis des Autors noch struktu- 
Tab. 3: Randomisierte kontrollierte klinische Rehabilitationsstudien zur Outcome-Verbesserung von Patienten mit schweren Bewusstseinsstörungen

\begin{tabular}{|c|c|c|c|c|c|}
\hline Intervention & Patienten & Krankheitsphase ${ }^{1}$ & Dosis Intervention & Ergebnis & Referenz \\
\hline $\begin{array}{l}\text { Vertikalisierung mittels } \\
\text { Kipptisch }\left(\text { Erigo }^{\circledR}\right) \\
\text { ohne versus mit robo- } \\
\text { tischem Beintraining }\end{array}$ & $\begin{array}{l}\text { Nur Kipptisch } n=22 \\
\text { Kipptisch + Beintraining: } \\
n=22 \\
\text { Ätiologie: SHT und nicht-SHT }\end{array}$ & 1-6 Monate & $\begin{array}{l}10 \text { Stunden innerhalb von } \\
3 \text { Wochen }\end{array}$ & $\begin{array}{l}\text { Kein Unterschied in CRS-R } \\
\text { zwischen Gruppen bei Follow- } \\
\text { up nach } 3 \text { Wochen }\end{array}$ & Krewer et al. 2015 \\
\hline $\begin{array}{l}\text { Familiar Auditory Sensory } \\
\text { Training (FAST; von Angehö- } \\
\text { rigen aufgenommene und } \\
\text { abgespielte Geschichten) } \\
\text { versus Placebo (Ruhe) }\end{array}$ & $\begin{array}{l}\text { FAST } n=8 \\
\text { Placebo } n=7 \\
\text { Ätiologie: nur SHT }\end{array}$ & 1-6 Monate & 40 Minuten/Tag für 42 Tage & $\begin{array}{l}\text { Besserung in CNC-Komaskala } \\
\text { in FAST-Gruppe; FAST mit mehr } \\
\text { Aktivierung im fMrt }\end{array}$ & Pape et al. 2015 \\
\hline $\begin{array}{l}\text { Anodale tDCS vs. Sham-Sti- } \\
\text { mulation über dem dorsola- } \\
\text { teralen präfrontalen Cortex } \\
\text { (Crossover-Design) }\end{array}$ & $\begin{array}{l}\text { Pat. in SRW } n=25 \\
\text { Pat. in MCS } n=30 \\
\text { Ätiologie: SHT und nicht-SHT }\end{array}$ & 2-5 Jahre & $\begin{array}{l}\text { Einmalige Applikation von } \\
\text { tDCS/Sham für } 20 \text { Minuten }\end{array}$ & $\begin{array}{l}\text { Nur bei MCS-Pat. transiente } \\
\text { Besserung in CRS-R }\end{array}$ & Thibaut et al. 2014 \\
\hline $\begin{array}{l}200-400 \text { mg Amantadin vs. } \\
\text { Placebo }\end{array}$ & $\begin{array}{l}\text { Amantadin } n=87 \\
\text { Placebo } n=97 \\
\text { Ätiologie: } \text { nur SHT }\end{array}$ & 1-4 Monate & Tägliche Gabe für 4 Wochen & $\begin{array}{l}\text { Schnellere Verbesserung in } \\
\text { DRS; kein Unterschied nach } 2 \\
\text { Follow-up }\end{array}$ & Giacino et al. 2012 \\
\hline $\begin{array}{l}10 \text { mg Zolpidem vs. Placebo } \\
\text { (Crossover-Design) }\end{array}$ & $\begin{array}{l}\mathrm{n}=15 \\
\text { Ätiologie: SHT und nicht-SHT }\end{array}$ & 1-278 Monate & Einmalige Gabe & $\begin{array}{l}\text { 1/15 Patienten mit Verbesse- } \\
\text { rung von SRW zu SMB/MCS }\end{array}$ & Whyte et al.2009 \\
\hline $\begin{array}{l}10 \text { mg Zolpidem open-label, } \\
\text { ggf. gefolgt von randomisier- } \\
\text { ter kontrollierter Gabe }\end{array}$ & $\begin{array}{l}\mathrm{n}=60 \\
\text { Ätiologie: SHT und nicht-SHT }\end{array}$ & 1 Monat-26 Jahre & Einmalige Gabe & $\begin{array}{l}4 / 60 \text { Patienten }(6,7 \%) \text { mit } \\
\text { Verbesserung in CRS-R, jedoch } \\
\text { in keinem Fall Verbesserung in } \\
\text { der Bewusstseinsdiagnose }\end{array}$ & Thonnardet al. 2013 \\
\hline
\end{tabular}

${ }^{1}$ Latenz zwischen akuter Hirnschädigung und Studieneinschluss

CNC = Coma Near Coma Scale; CRS-R = Coma Recovery Scale - Revised; DRS = Disability Rating Scale; WNSSP = Western Neuro Sensory Stimulation Profile

rierte Metaanalysen auf hohem methodischen Niveau, wenngleich sich mehrere Übersichtsartikel dieses Themas bereits angenommen haben $[31,56]$.

Die im Rahmen von klinischen Studien untersuchten therapeutischen Maßnahmen unterteilen sich in Pharmakotherapie (i.b. Amantadin und Zolpidem), elektrische Stimulationsverfahren (i.b. transkranielle Gleichstromstimulation, tDCS), sensorische Stimulationsverfahren sowie Vertikalisierung mit oder ohne robotischem Beintraining (Tab. 3) [29, 53, 73, 95, 97, 105].

Die bisher methodisch aufwendigste und aussagefähigste Studie untersuchte den Effekt einer vierwöchigen Amantadin-Therapie (200-400mg) im Rahmen einer randomisierten, plazebokontrollierten multizentrischen Studie mit 184 SHT-Patienten [29]. Amantadin ist ein NMDA-Rezeptor-Antagonist mit zusätzlicher dopaminerger Wirkungskomponente. Bei dieser Studie zeigte sich eine höhere Besserungsrate in der Amantadin-Gruppe in den Werten der Disability Rating Scale am Ende der vierwöchigen Intervention. Nach weiteren zwei Wochen ohne Gabe der Studienmedikation waren diese Vorteile der Verum-Gruppe nicht mehr nachweisbar, da nun die Kontrollgruppe »aufgeholt« hatte und die Erholungskurve der Amantadin-Gruppe abflachte. Dies sollte meines Erachtens jedoch nicht negativ interpretiert werden, sondern vielmehr als weiterer Beleg für die Wirksamkeit von Amantadin. Die klinische Konsequenz sollte sein, dass Amantadin unter Beachtung der Kontraindikationen systematisch bei allen SHT-Patienten der NNFR im SRW oder SMB/MCS eingesetzt werden sollte und bei positiven Effekten auch für Zeiträume von länger als vier Wochen. Die längerfristige Gabe scheint auch deswegen realistisch, weil sich in dieser randomisierten klinischen Studie keine erhöhten Nebenwirkungsraten im Vergleich zu Plazebo zeigten. Auch wenn dies nicht evidenzbasiert ist, so scheint uns in Analogie zu diesen Ergebnissen die Verwendung von Amantadin bei nicht-traumatischer Genese der Hirnschädigung sinnvoll zu sein.

Ein paradoxer wachheits- und kontaktfähigkeits-steigernder Effekt des Schlafmittels Zolpidem wurde mehrfach anekdotisch in der Literatur beschrieben [14]. Die entsprechenden randomisierten klinischen Studien hierzu zeigten eher geringe Effekte bei einem geringen Prozentsatz der Patienten (Tab. 3). Es scheinen vor allem SMB/ MCS-Patienten zu sein, die durch Zolpidem mehr Aktivität und Kontaktfähigkeit erreichen und weniger SRW-Patienten, die hierdurch primär kontaktfähig würden.

Auch wachheitssteigernde Effekte von Apomorphin, einem starken Dopaminagonisten und intrathekalem Baclofen wurden mehrfach berichtet, allerdings bisher nur in Form von Einzelfallberichten oder kleinen Fallserien [23, 76]. Die Effekte anderer aufgrund ihres Wirkprofils potenziell sinnvoller Stimulanzien wurden bisher kaum wissenschaftlich untersucht [67]. Im klinischen Alltag beobachten wir gelegentlich positive Effekte unter Modafinil, Levodopa, Serotonin-Wiederaufnahmehemmern oder Methylphenidat.

Eine weitere Therapieoption, die zunehmend ins Interesse der Rehabilitationswissenschaft rückt, ist die Musiktherapie, die bereits in kleineren Serien Effekte hin zu mehr Wachheit, mehr Kontaktfähigkeit und zur posi- 
tiven Veränderung von elektrophysiologischen Markern von Wachheit und Hirnaktivität gezeigt hat [7, 64, 84]. Es spiegelt auch die Erfahrung des Autors wider, dass die Musiktherapie gerade in sehr frühen Krankheitsphasen Wachheit und Kontaktfähigkeit fördern kann und andererseits auch einen klar symptomatisch beruhigenden Effekt bei Patienten mit vegetativer Dysregulation hat.

Bei SMB/MCS-Patienten stellt sich häufig die Frage, ob durch technische Lösungen zumindest der Aufbau eines stabilen Kommunikationskanals möglich ist. Da in den allermeisten Fällen bei SMB/MCS-Patienten die Feinmotorik der Extremitäten nicht zur manuellen Bedienung eines elektronischen Kommunikationssystems ausreicht, kann prinzipiell der Einsatz eines Systems erwogen werden, das durch Augensteuerung bedient wird. Nach Kenntnisstand des Autors liegen auch hierzu keine klinischen Studien vor. Allerdings haben wir bisher nur wenige positive Erfahrungen bei SMB/MCS-Patienten gesammelt. Dies mag daran liegen, dass häufig die Aufmerksamkeitsspanne und Belastbarkeit der Patienten nicht ausreicht, um solche System zuverlässig mit den Augen steuern zu können. Eine bisher rein experimentelle Option ist die Steuerung von Brain-Computer-Interfaces mittels ereigniskorrelierter Potenziale unter Verwendung spezieller kognitiver Aufgaben zur Ja-/Nein-Kommunikation [62]. Dies gelang bisher jedoch nur bei einem geringen Prozentsatz der SMB/ MCS-Patienten in einem rein wissenschaftlichen Setting.

Auch wenn es somit bisher nur sehr wenige evidenzbasierte Therapien zur Verbesserung des Bewusstseinszustandes für Patienten im Koma, SRW oder SMB/ MCS gibt, so ist es in der NNFR umso wichtiger, die häufig auftretenden Komplikationen rasch und konsequent zu behandeln.

\section{Häufige Komplikationen}

Patienten der NNFR mit schweren Bewusstseinsstörungen haben ein hohes Risiko, Komplikationen zu erleiden. In einer prospektiven Studie wird es mit einer Rate von 0,4 Komplikationen pro SHT-Patient und Behandlungswoche angegeben [106]. In dieser Studie nahm die Wahrscheinlichkeit für Komplikationen mit zunehmender Aufenthaltsdauer ab. Im Laufe der 6-wöchigen prospektiven Follow-up-Phase entwickelten $83 \%$ der Patienten mindestens eine und durchschnittlich zwei bis drei Komplikationen. Am häufigsten traten Spastik, Agitiertheit/aggressives Verhalten, Harnwegsinfekte, Störungen des Tag-Nacht-Rhythmus und Erbrechen auf. Pneumonien waren hingegen mit nur 3\% aller dokumentierten Komplikationen überraschend selten. Andere spezifisch neurologische Komplikationen wie z. B. epileptische Anfälle oder Entwicklung eines Hydrozephalus waren ebenfalls relativ selten.

In einer früheren Studie mit einer entsprechenden Patientenpopulation wiesen $49 \%$ der Patienten min- destens eine Episode mit Fieber auf, wobei auch hier Harnwegsinfekte häufiger waren als Pneumonien [71].

Ein etwas anderes Komplikationsprofil zeigt eine Studie mit einem gemischt traumatischen wie auch nicht-traumatischen Patientengut mit schweren Bewusstseinsstörungen [24]. Von diesen 68 Patienten entwickelten $62 \%$ eine Pneumonie, 57\% eine Spastik, $47 \%$ einen Harnwegsinfekt, 46\% einen epileptischen Anfall und 38\% einen Hydrozephalus. Die Anzahl der Komplikationen pro Patient korrelierte dabei klar mit dem funktionellen Patientenstatus nach einem Jahr. In einer weiteren Studie wiesen 89\% der Patienten im SRW oder SMB/MCS eine zumindest leichtgradige und 62\% eine schwere Spastik auf [96]. Andere Daten erbrachten Dekubitus-Raten von $14 \%$ und bei tiefen Beinvenenthrombosen von $17 \%$ [89].

Nach unserer Erfahrung stellen eine vegetative Instabilität und Dysregulation, die Behandlung eines therapierefraktären Status epilepticus insbesondere bei HIE-Patienten wie auch eine schwere Tetraspastik die größten therapeutischen Herausforderungen in dieser Patientenpopulation in der NNFR dar. Die interdisziplinären Behandlungsteams sollten hierfür standardisierte Behandlungskonzepte formulieren und konsequent anwenden. Für das Beispiel der multimodalen Spastiktherapie umfassen solche Konzepte ein breites Spektrum von der oralen Antispastikagabe über die lokale Behandlung mit Botulinum Neurotoxin A, redressierende Verbände bis hin zur intrathekalen BaclofenpumpenImplantation sowie ggf. weiteren neuroorthopädischen Operationen [89].

Dieses Know-how zur Behandlung und zum Management dieser schweren Komplikationen ist ein wesentliches Alleinstellungsmerkmal der Kliniken der NNFR.

\section{Langzeitverlauf und Prognostizierung}

Mehrere Studien haben in den vergangenen Jahren das Langzeitbehandlungsergebnis von Patienten mit schweren Bewusstseinsstörungen nach akuter Hirnschädigung untersucht und prognostische Faktoren identifiziert [37, 40, 48-50, 68]. Hierbei geht es in dieser Übersichtsarbeit explizit nicht um prognostische Faktoren in der Frühphase, d.h. innerhalb der ersten Tage und Wochen auf der Intensivstation. Diese Aspekte würden den Rahmen dieser Arbeit sprengen, so dass wir hier nur auf aktuelle Übersichtsarbeiten und Leitlinien verweisen können $[35,58,70]$. An dieser Stelle werden hingegen diejenigen Prognosefaktoren zusammengefasst, die typischerweise während der NNFR erhoben werden. Tabelle 4 gibt einen Überblick über konsistent berichtete Prognosefaktoren bei Aufnahme in die NNFR und jenseits eines rein experimentellen Settings in einem Bildgebungszentrum oder elektrophysiologischem Labor. Tatsächlich gibt es nur wenige Daten darüber, welche prognostische Aussagekraft z. B. Neuronen-spezifische Enolase, somatosensibel 
Tab. 4: Prognosefaktoren in der Aufnahmesituation in die neurologisch-neurochirurgische Frührehabilitation

\begin{tabular}{l|l}
\hline \multicolumn{1}{c|}{ Günstige Prognosefaktoren } & \multicolumn{1}{c}{ Ungünstige Prognosefaktoren } \\
\hline niedriges Alter & höheres Alter \\
SMB/MCS (höhere CRS-R-Werte) bei Aufnahme in die NNFR & SRW (niedrigere CRS-R-Werte) bei Aufnahme in die NNFR \\
traumatische Ätiologie & nicht-traumatische Ätiologie \\
& Notwendigkeit eines VP-Shunts (nur SHT) \\
& Z.n. dekompressiver Kraniektomie (nur SHT) \\
\hline
\end{tabular}

CRS-R = Coma Recovery Scale-Revised; NNFR = neurologisch-neurochirurgische Frührehabilitation; SHT = Schädel-Hirn-Trauma; SMB/MCS = Syndrom

des minimalen Bewusstseins/Minimally Conscious State; SRW = Syndrom der reaktionslosen Wachheit; VP-Shunt = Ventrikulo-peritonealer Shunt

evozierte Potenziale (SEP) oder Elektroenzephalogramm (EEG) haben, wenn sie jenseits der ersten Tage auf der Intensivstation erhoben werden. Für die SEP konnte z.B. gezeigt werden, dass ursprünglich bilateral ausgefallene kortikale Reizantworten (N2O) - ein prognostisch sehr ungünstiges Zeichen bei HIE - sich im Laufe der NNFR bei bis $\mathrm{zu}$ 50\% der Patienten wieder erholen können [86]. Insbesondere bei SHT-Patienten war das Wiederauftreten der kortikalen Reizantworten mit einer auch funktionell relevanten klinischen Erholung assoziiert, was auch unseren eigenen Daten entspricht [50]. Selbst bei HIE-Patienten ist der einmalig dokumentierte bilaterale Ausfall der SEP-N2O nicht zwingend und in jedem Fall mit einer infausten Prognose verbunden. Wir konnten zeigen, dass die Falsch-Positiv-Rate für die Vorhersage einer ungünstigen Prognose, je nach Verwendung der Definition für ein günstiges Outcome (funktionelle Teilerholung vs. Wiedererlangen der Kommunikationsfähigkeit) und Zeitpunkt der SEP-Ableitung (Intensivstation vs. in der NNFR) selbst für HIE-Patienten zwischen $14 \%$ und 29\% liegt [40]. Das bedeutet, dass bei alleiniger Betrachtung der SEP-Ergebnisse ca. ein Fünftel der Patienten ein gutes Outcome erreicht, obwohl die fehlenden bilateralen kortikalen Reizantworten nach gängiger Lehrmeinung dies gänzlich ausschließen würden.

Ein weiterer wichtiger prognostischer Faktor scheint das Patientenalter zu sein. Sowohl bei HIE- als auch bei SHT-Patienten ist ein höheres Alter ein unabhängiger Prädiktor für ein ungünstigeres Outcome, und zwar sowohl für das Wiedererlangen des Bewusstseins als auch das Erreichen einer funktionellen Teilunabhängigkeit $[40,50]$. In unserer eigenen HIE-Serie war der älteste Patient, der noch ein gutes funktionelles Outcome erreichen konnte, nachdem er im SRW bzw. SMB/MCS in die NNFR aufgenommen worden war, 50 Jahre alt [40].

In einer retrospektiven Analyse von 36 Patienten, die bei Aufnahme zur stationären Rehabilitationsbehandlung mindestens 4 Wochen im SRW oder SMB/MCS gewesen waren, überwanden $69 \%$ innerhalb von einem Jahr das SMB/MCS hin zu mehr Kontaktfähigkeit, wobei traumatische Patienten eine höhere Wahrscheinlichkeit aufwiesen als Patienten mit nicht-traumatischer Genese (77\% vs. 57\%) [48]. Die durchschnittliche Dauer bis zum Überwinden des SMB/MCS betrug 9 Wochen. Die Wahrscheinlichkeit, das SMB/MCS zu überwinden, war höher, wenn die Patienten bei Aufnahme im SMB/MCS waren im Vergleich zum SRW bei Aufnahme (80\% vs. 45\%). Im Langzeitverlauf von 1-4 Jahren nach Hirnschädigung konnten $43 \%$ der Überlebenden wieder mindestens 8 Stunden pro Tag alleine zu Hause zurechtkommen, und $22 \%$ konnten wieder in Arbeitsmarkt bzw. Schule reintegriert werden. Im Verlauf zwischen einem und vier Jahren waren weitere Funktionszugewinne $\mathrm{zu}$ beobachten. Betont werden muss bei dieser Studie, dass sich die Wahrscheinlichkeiten nur auf die Überlebenden Patienten bezieht, d.h. die verstorbenen Patienten oder die ohne weitere Studienvisite bleiben unberücksichtigt. Dies entspricht aber häufig nicht der alltäglichen Beratungssituation in der NNFR, in der die Angehörigen Informationen über das zu erwartenden Behandlungsergebnis am Ende der stationären Rehabilitation bzw. nach einem Jahr erwarten. Um hier für eine gute Beratung bzw. "Expectation Management« Daten zur Verfügung zu stellen, haben wir die Literatur analysiert, um mehrere Kernfragen beantworten zu können (Tab. 5). Diese Kernfragen lauten aus Sicht des Autors:

Wenn man bei Aufnahme in die NNFR im Koma, SRW oder SMB/MCS ist, wie groß ist dann die Wahrscheinlichkeit ...

- am Ende der NNFR (und ggf. weiterer Phasen) das MCS überwunden $\mathrm{zu}$ haben?

am Ende der NNFR (und ggf. weiterer Phasen) wieder funktionell teilunabhängig zu sein?

nach einem Jahr das MCS überwunden zu haben?

nach einem Jahr wieder funktionell teilunabhängig zu sein?

nach einem Jahr wieder funktionell komplett unabhängig zu sein?

nach mehr als einem Jahr im SRW noch das SMB/MCS zu erreichen?

nach mehr als einem Jahr noch das SMB/MCS zu überwinden?

Leider ist die Datenbasis zur Beantwortung dieser Fragen dünn, und viele Daten sind nicht vergleichbar, denn es wurden unterschiedliche Ein- und Ausschlusskriterien sowie Analysestrategien verwendet. Wir untersuchten in verschiedenen Patientenpopulationen die Dynamik des Rehabilitationsverlaufes. Während von 188 SHT-Patienten im SRW bzw. SMB/MCS am Ende der NNFR 37\% wieder das SMB/MCS überwunden hatten, betrug dieser 


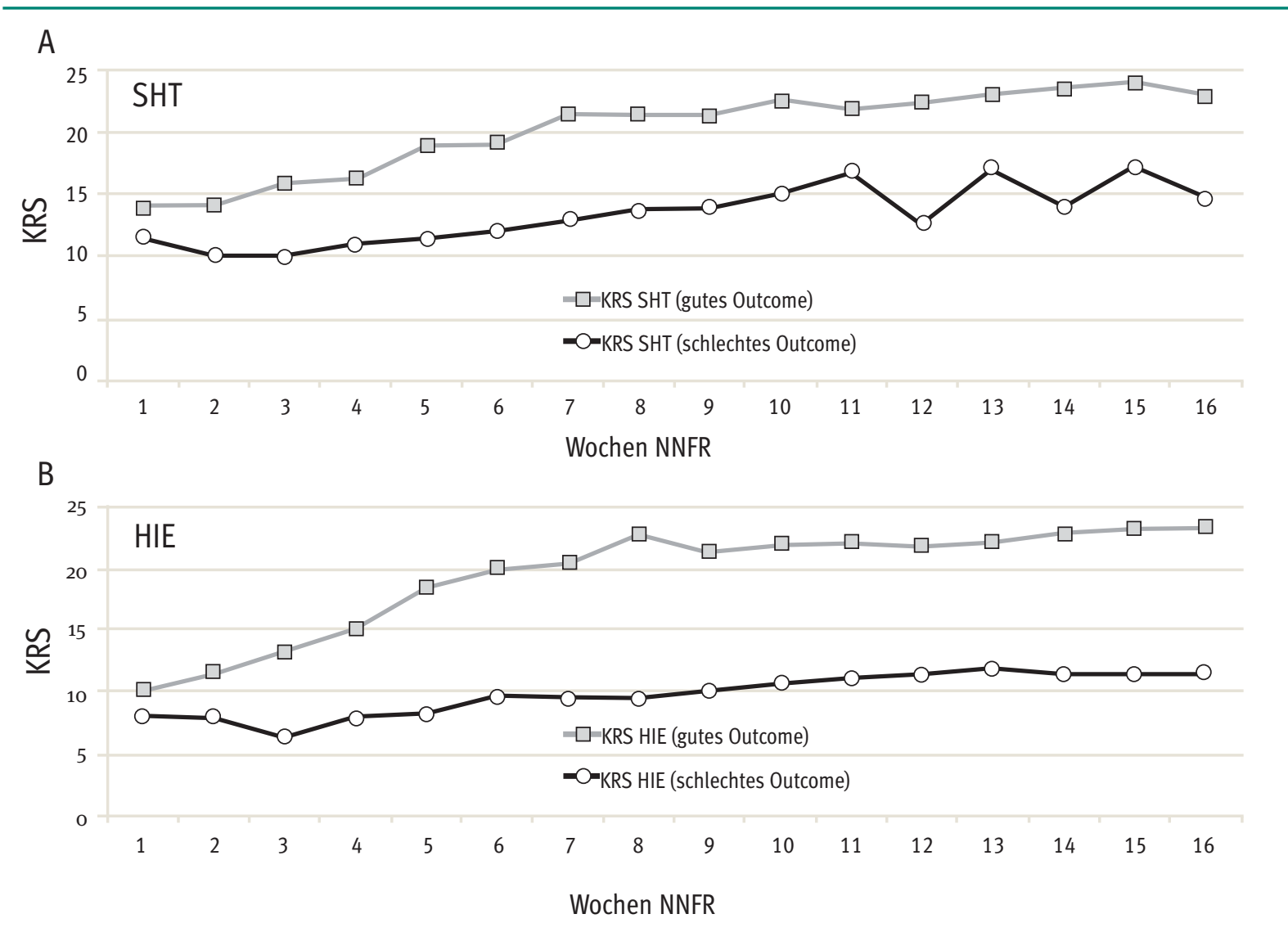

Abb. 4: Zeitverlauf des Wiedererlangen des Bewusstseins, gemessen mit der Koma Remissions Skala (KRS) im Verlauf der neurologisch-neurochirurgischen Frührehabilitation bei Patienten mit Schädel-Hirn-Trauma (SHT; A) bzw. Hypoxisch-Ischämischer Enzephalopathie (HIE; B). Die Patienten wurden in eine Gruppe mit gutem (hellgraue Linie und Quadrate) bzw. »schlechtem « (schwarze Linie mit weißen Kreisen) Bewusstseins-Outcome unterteilt (gut: KRS > 22; schlecht: KRS $\leq 22$ ). Abbildung basierend auf den Originaldaten der Publikationen [40, 50]

Anteil in der Gruppe der 113 Patienten mit HIE nur $20 \%$ $[40,50]$. Dies entspricht der seit langem bekannten Tatsache, dass Patienten mit traumatischer Genese generell eine bessere Prognose haben als solche mit einer nicht-traumatischen Genese [79]. Dieser Unterschied zeigte sich auch bei Betrachtung der Patientenquote, die wieder zumindest eine funktionelle Teilunabhängigkeit erreicht hatten $(17 \%$ der traumatischen und $6 \%$ der HIEPatienten) [40, 50]. Auch die Zeitverläufe während der NNFR sind informativ bezüglich der Abschätzung des

Tab. 5: Wahrscheinlichkeiten für verschiedene Outcome-Szenarien bei Patienten, die bei Aufnahme in die NNFR im Koma, SRW oder SMB/ MCS waren

\begin{tabular}{|c|c|c|}
\hline Wahrscheinlichkeit für & traumatische Genese & nicht-traumatische Genese \\
\hline am Ende NNFR besser als SMB/MCS & $\begin{array}{l}68 \%[68] \\
37 \%[50]\end{array}$ & $\begin{array}{l}20 \% \text { (HIE) [40] } \\
18 \% \text { (HIE) [37] } \\
40 \% \text { (SAB) [49] }\end{array}$ \\
\hline am Ende NNFR mind. funktionell teilunabhängig & $\begin{array}{l}23 \%[68] \\
17 \%[50]\end{array}$ & $\begin{array}{l}6 \% \text { (HIE) [40] } \\
19 \% \text { (SAB) [49] }\end{array}$ \\
\hline nach 1 Jahr besser als SMB/MCS & KOPFregister [33] & KOPFregister / HOPE $[33,59]$ \\
\hline nach 1 Jahr funktionell teilunabhängig & KOPFregister [33] & KOPFregister / HOPE $[33,59]$ \\
\hline nach 1 Jahr komplett unabhängig & KOPFregister [33] & KOPFregister / HOPE $[33,59]$ \\
\hline $\begin{array}{l}\text { Wahrscheinlichkeit, nach mehr als } 1 \text { Jahr noch } \\
\text { das SMB/MCS zu überwinden }\end{array}$ & $\begin{array}{l}50 \% \text {; alle vorher SMB/MCS [61] } \\
28 \% \text {; alle vorher SRW [20] }\end{array}$ & $\begin{array}{l}22 \% \text {; alle vorher SMB/MCS [61] } \\
6 \% \text {; alle vorher SRW [20] }\end{array}$ \\
\hline $\begin{array}{l}\text { Wahrscheinlichkeit, nach mehr als } 1 \text { Jahr SRW } \\
\text { noch mind. SMB/MCS zu erreichen }\end{array}$ & $\begin{array}{l}44 \%[20] \\
0 \%([61]\end{array}$ & $\begin{array}{l}\text { 13\%; alle vorher SRW [20] } \\
0 \%[61]\end{array}$ \\
\hline
\end{tabular}

HIE = Hypoxisch-Ischämische Enzephalopathie; NNFR = Neurologisch-Neurochirurgische Frührehabilitation; SAB = Subarachnoidalblutung; SMB/MCS = Syndrom des Minimalbewusstseins/Minimally Conscious State; SRW = Syndrom der reaktionslosen Wachheit 


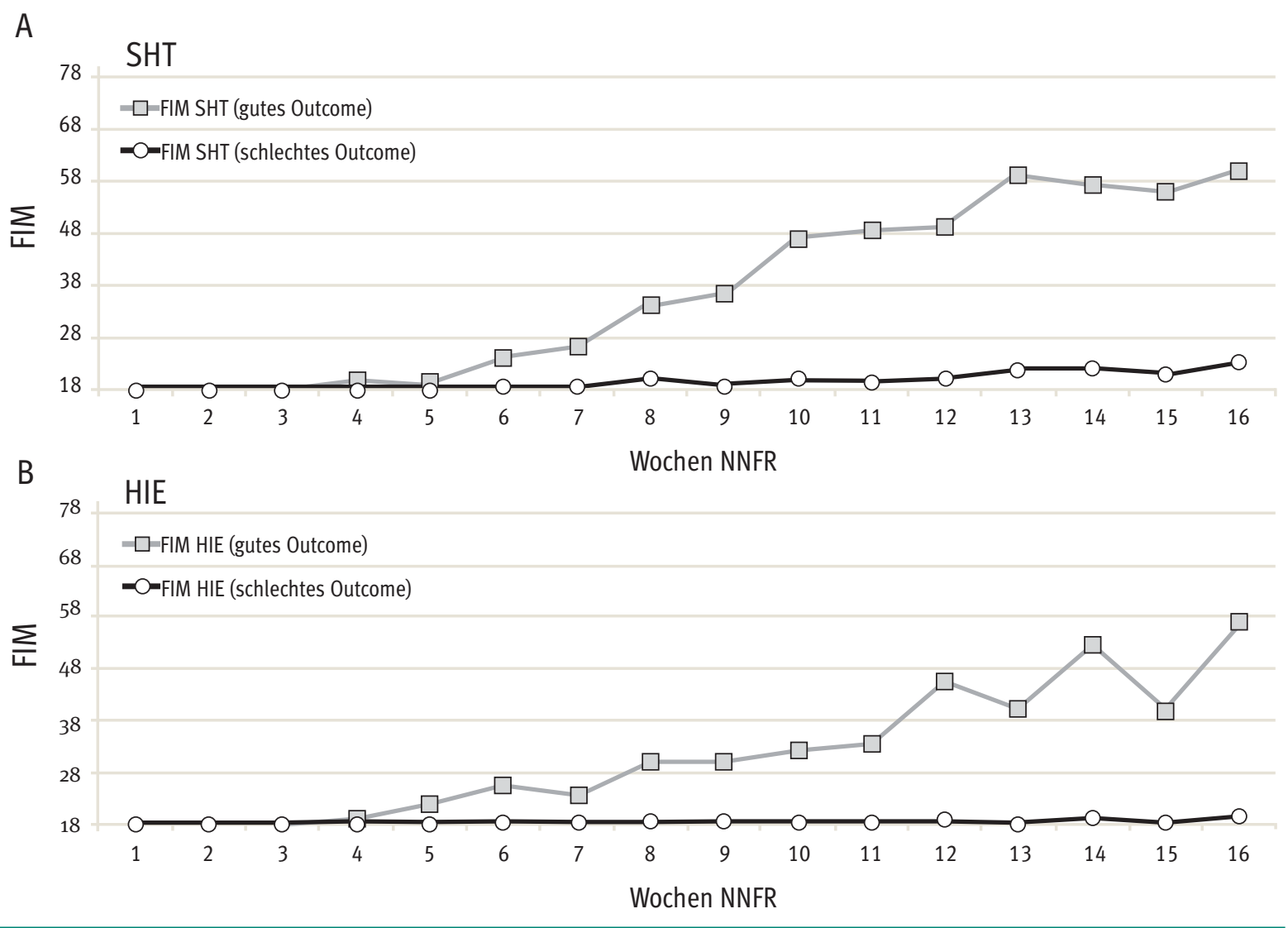

Abb. 5: Zeitverlauf der Verbesserung in den Aktivitäten des täglichen Lebens, gemessen mit der Functional Independence Measure (FIM) im Verlauf der neurologisch-neurochirurgischen Frührehabilitation (NNFR) bei Patienten mit Schädel-Hirn-Trauma (SHT; A) bzw. HypoxischIschämischer Enzephalopathie (HIE; B). Die Patienten wurden in eine Gruppe mit gutem (hellgraue Linie und Quadrate) bzw. "schlechtem« (schwarze Linie mit weißen Kreisen) funktionellem Outcome unterteilt (gut: FIM > 30; schlecht: FIM $\leq 30$ ). Beachten Sie, dass die "gute« Gruppe mit erreichen einer zumindest funktionellen Teilunabhängigkeit im Laufe der NNFR bei beiden Diagnosen nach ca. 4-6 Wochen eine positive Dynamik aufweist und sich von der ungünstigen Outcome-Gruppe separiert. Abbildung basierend auf den Originaldaten der Publikationen $[40,50]$

weiteren Rehabilitationsverlaufs (Abb. 4, Abb. 5). Sowohl beim klinischen Verlauf der Bewusstseinszustandes (Abb. 4) als auch beim Verlauf der Selbstständigkeit in den Tagesaktivitäten (Abb. 5; gemessen mit dem FIM) kann früh im Rehabilitationsverlauf eine Gruppe mit günstigerem von einer mit ungünstigerem Behandlungsergebnis am Ende der NNFR unterschieden werden. Bei den HIEPatienten betrug die größte Latenz bis zum Beginn einer messbaren KRS-Verbesserung, die später im Erreichen einer normalen Kontakt- und Kommunikationsfähigkeit resultierte, 10-12 Wochen [40]. Diese Beobachtungsphase, die notwendig war, um keines der positiven Outcomes $\mathrm{zu}$ »verpassen«, liegt interessanterweise oberhalb der aktuellen durchschnittlichen Verweildauer in der NNFR von 8 Wochen [78].

Prospektive Daten über das Behandlungsergebnis nach einem Jahr sind bisher kaum verfügbar. Hier werden zwei multizentrische prospektive Beobachtungsstudien in absehbarer Zeit Ergebnisse liefern (KOPFregister und Projekt HOPE) [33, 59].

In einer kleineren prospektiven Serie mit 31 SRWPatienten (SHT n=17, nicht-SHT n=14) ca. 6 Wochen nach akuter Hirnschädigung hatten nach 1 Jahr insgesamt $68 \%$ der Patienten zumindest das SMB/MCS wieder erreicht (77\% der SHT-Patienten und 57\% der NichtSHT-Patienten) [4]. Nur einer der Patienten überwand auch das SMB/MCS und zeigte funktionelle Kommunikation und Objektgebrauch (traumatische Genese). Die allermeisten Verbesserungen ergaben sich in den ersten Monaten der Beobachtungsphase.

Zwei Studien mit relativ kleiner Patientenzahl konnten vor einigen Jahren zeigen, dass es tatsächlich keine begrenzten Zeitfenster für das Wiedererlangen des Bewusstseins mit funktioneller Kommunikationsfähigkeit gibt, so wie in früheren Studien und Empfehlungen postuliert [79]. In beiden Studien wurden SRW- und SMB/ MCS-Patienten für mehrere Jahre nachuntersucht und $12 \%$ bzw. $25 \%$ überwanden im Verlauf das SMB/MCS [20, 61]. Dabei zeigte sich jedoch, dass auch diese "positiven" Verläufe im mehrjährigen Beobachtungszeitraum nicht zu einer funktionellen Unabhängigkeit in den Aktivitäten des täglichen Lebens (ATL) führte und dass in allen Fällen teilweise erhebliche Behinderungen und kognitive Einschränkungen auf Dauer fortbestanden [21]. 
Severe disorders of consciousness in early neurological and neurosurgical rehabilitation

A. Bender

\section{Summary}

Severe disorders of consciousness (DOC) due to an acute brain injury clinically manifest as either coma, unresponsive wakefulness syndrome (UWS; old terminology: vegetative state), or minimally conscious state (MCS). DOC affect a substantial proportion of patients treated in specialized early neurorehabilitation inpatient centers in Germany. Patients with DOC have several distinct needs and thus pose special challenges for the therapeutic teams in the neurorehabilitation facilities. They range from the correct diagnosis of consciousness (e.g., by means of the Coma Recovery ScaleRevised; CRS-R), management of frequent medical complications, analysis of prognostic factors, and interdisciplinary therapeutic measures to counseling relatives and discussing questions about limitations or withdrawal from life support. Also, recent developments in modern coma research may substantially contribute to establishing the correct diagnosis or prognosis, e.g., by means of functional magnetic resonance imaging (fMRI) or electrophysiological analysis of event-related potentials. Yet, they may also lead to a degree of uncertainty among relatives and family caregivers.

This review article intends to provide an overview of these different clinically relevant aspects of managing DOC patients in inpatient neurorehabilitation.

Keywords: coma, unresponsive wakefulness syndrome, minimally conscious state, prognosis, coma research

Neurol Rehabil 2016; 22(3): 192-208

(C) Hippocampus Verlag 2016

Ein »gutes Outcome« muss angesichts einer schwerwiegenden Hirnschädigung mit vielen Monaten im Koma oder SRW nicht zwingend definiert werden als eine weitestgehende Wiederherstellung ohne oder nur mit geringen Alltagseinschränkungen, so wie es z.B. in vielen Schlaganfallstudien normal ist. Auch das Wiedererreichen einer Integration in das häusliche Umfeld mit Teilhabe am Familienleben und Kommunikationsfähigkeit kann individuell als positives Behandlungsergebnis wahrgenommen werden, auch wenn natürlich hieraus eine enorme psychische, körperliche wie auch sozioökonomische Belastung für die Familien resultieren kann [104].

\section{Medizinethische Aspekte}

Die Betreuung von Patienten mit schweren Bewusstseinsstörungen in der NNFR streift in vielen Fällen unweigerlich medizinethische wie auch medizinjuristische Fragen [45]. Dabei geht es häufig darum, wie lange und wie intensiv eine Behandlung fortgesetzt werden sollte angesichts der $\mathrm{zu}$ erwartenden schweren Hirnschädigung mit dauerhaften, schwerwiegenden Behinderungen [44]. Selten resultieren hieraus gerichtliche Auseinandersetzungen mit hoher medialer Beachtung [12]. Bei der Angehörigenberatung ist es dabei von großer Bedeutung eine realistische Erwartungshaltung herbei zu führen. Welche Erwartung an den Heilungsverlauf ist noch realistisch, welche nicht mehr (s. oben)?
Die Basis einer jeden medizinischen Behandlung stellen die bestehende ärztliche Indikation einerseits und die Einwilligung der Patienten bzw. ihrer gesetzlichen Betreuer andererseits dar. Entfällt eine dieser beiden Voraussetzungen handelt es sich bei dennoch fortgesetzter Behandlung ggf. um Körperverletzung. Schwierig ist dabei allerdings die Frage, wie lange eine Therapie noch indiziert ist. Rechtfertigt das Rehabilitationsziel der Pflegbarkeit im häuslichen Umfeld bei fortbestehendem SRW die fortgesetzte Therapie, auch wenn eine funktionelle Erholung des Patienten nicht mehr realistisch ist? Diese Frage muss individuell und idealerweise im Einklang mit den betreuenden Angehörigen gelöst werden. Maßgebend ist hierbei der Patientenwille, ggf. auch der mutmaßliche [45]. Hilfreich ist nach der Erfahrung des Autors der Aspekt der relativen Entschleunigung gegenüber der Initialphase auf den Intensivstationen der Akutkliniken. Die Beobachtung des Krankheitsverlaufs in den ersten Wochen der NNFR liefert dabei sowohl für die meisten Angehörigen wie auch das professionelle Behandlungsteam wichtige Hinweise für die realistische Einschätzung des weiteren Outcomes. Wir konnten zeigen, dass Angehörige von Patienten mit schweren Bewusstseinsstörungen in der NNFR den Bewusstseinsgrad der Betroffenen durchaus realistisch einschätzen konnten [43]. 59\% der Angehörigen hatten bereits über Therapielimitierungen nachgedacht oder diese gemeinsam mit dem Behandlungsteam vereinbart.

Schwierig ist dabei der offensichtlich bei vielen Patienten stattfindende Perspektivenwechsel, der mit Eintritt einer schweren Behinderung im Vergleich zum normalen vorherigen Gesundheitszustand eintreten kann. Anders ist es wohl nicht zu erklären, dass ein relevanter Anteil von LIS-Patienten trotz der nahezu maximalen Behinderung eine relativ normale Lebensqualität angibt [10, 63]. Auch wenn das LIS keine Bewusstseinsstörung ist, so zeigt dieser Vergleich dennoch, dass Vorsicht und ein wenig Demut bei der Beurteilung des mutmaßlichen Patientenwillens aus der Perspektive des Gesunden geboten ist.

Sollte man jedoch $\mathrm{zu}$ der Einsicht gelangen, dass eine Behandlung nicht mehr indiziert bzw. gegen den expliziten oder mutmaßlichen Patientenwillen verstößt, muss sie beendet und eine Therapiezieländerung im Sinne einer palliativen Therapie vorgenommen werden [13]. Während viele Neurologen in der entsprechenden Situation einer Therapielimitierung im Sinne von z.B. »Do not resuscitate« nicht mehr intubieren und beatmen oder keiner Hämofiltration/Dialyse zustimmen würden, gibt es offensichtlich wesentlich mehr Vorbehalte beim Einstellen von Ernährungs- und Flüssigkeitstherapie [55].

Zunehmend erschwert wird dieses Themengebiet noch durch die Erkenntnisse der modernen Komaforschung. Was bedeutet es, wenn Patienten, die sich klinisch im SRW befinden, im fMRT überzufällig häufig 
korrekt auf Fragen antworten können, indem sie einen Kommunikationscode benutzen, bei dem das Vorstellen von Tennisspielen als "Ja" und das gedankliche durch die Wohnung gehen als "Nein" verwendet werden [66]. So spannend und faszinierend fMRT, PET und ereigniskorrelierte Potenziale bei Patienten auch sein mögen, so sehr können sie auch die Unsicherheit der Angehörigen erhöhen [42, 74]. Dies gilt umso mehr, als diese Methoden noch weit weg sind von der klinischen Routineversorgung oder einer flächendeckenden Verfügbarkeit und noch viel methodische wie auch inhaltliche Unsicherheit bezüglich ihrer tatsächlichen Aussagekraft im Kontext der klinischen Standardversorgung besteht [74].

\section{Schlussfolgerungen}

Die Mitglieder der interdisziplinären Behandlungsteams der NNFR sollten mit den besonderen Herausforderungen der Betreuung von Patienten mit schweren Bewusstseinsstörungen vertraut sein. Diese Herausforderungen beginnen mit dem Stellen der richtigen Bewusstseinsdiagnose durch Verwendung standardisierter klinischer Skalen und reichen über das Erkennen und Behandeln komplizierender neurologischer und nicht-neurologischer Komplikationen bis hin zum realistischen Beraten verunsicherter Angehöriger. Die Beschleunigung des Wiedererlangens des Bewusstseins mit Amantadin ist derzeit noch der einzige Therapiebaustein des interdisziplinären Behandlungsansatzes, der eine gute Evidenz für seine Wirksamkeit besitzt. Weitere Therapiestudien hoher methodischer Qualität sind unbedingt notwendig, um dieses Forschungsfeld und die Qualität der Patientenversorgung weiter zu entwickeln. Vor einem zu unkritischen Einsatz von vermeintlich sicheren prognostischen Markern muss gewarnt werden, insbesondere wenn sie isoliert und nicht im Kontext aller zur Verfügung stehenden Informationen verwendet werden. In diesem Zusammenhang bieten auch die SEP keine so hohe Spezifität, dass sie alleinig für eine individuelle Therapieentscheidung verwendet werden könnten. Die Outcome- und Prognoseforschung hat in den letzten Jahren klar gezeigt, dass es keine starren Zeitfenster für ein Erholungspotenzial nach schwerer Hirnschädigung mit Bewusstseinsstörungen gibt. Langfristige Verbesserungsschritte sind möglich, wenngleich die Wahrscheinlichkeit eines günstigen funktionellen Behandlungsergebnisses mit Teilunabhängigkeit in den ATL mit zunehmender Dauer von Koma, SRW oder SMB/MCS deutlich sinkt. Dennoch kann es aus der Perspektive der betroffenen Patienten wie auch der Angehörigen gleichwohl subjektiv auch jenseits einer kompletten funktionellen Erholung ein positives Outcome geben, insbesondere dann z.B. wenn Patienten mit wiedererlangter Kommunikationsfähigkeit im häuslichen Setting integriert und am Familien- und Sozialleben teilhaben können.

\section{Abkürzungsverzeichnis}

\begin{tabular}{|l|l|}
\hline ARAS & Aufsteigendes retikuläres Aktivierungssystem \\
\hline ATL & Aktivitäten des täglichen Lebens \\
\hline CIP/CIM & Critical-Illness-Polyneuropathy/-Myopathie \\
\hline CRS-R & Coma Recovery Scale - Revised \\
\hline DOC & Disorders of consciousness \\
\hline EEG & Elektroenzephalogramm \\
\hline FIM & Functional Independence Measure \\
\hline fMRT/fMRI & Funktionelle Magnetresonanztomographie \\
\hline FOUR & Full Outline of Unresponsiveness-Skala \\
\hline GCS & Glasgow Coma Skala \\
\hline GOS & Glasgow Outcome Skala \\
\hline HIE & Hypoxisch-ischämische Enzephalopathie \\
\hline KRS & Koma Remissions Skala \\
\hline LIS & Locked-in-Syndrom \\
\hline MCS & Minimally Conscious State \\
\hline NKSE & Nicht-konvulsiver Status epilepticus \\
\hline NNFR & Neurologisch-neurochirurgische Frührehabilitation \\
\hline PET & Positronenemissionstomographie \\
\hline SAB & Subarachnoidalblutung \\
\hline SEP & Somatosensibel evozierte Potenziale \\
\hline SHT & Schädel-Hirn-Trauma \\
\hline SMART & Sensory Modality Assessment and Rehabilitation \\
\hline SMB & Syndrom des minimalen Bewusstseins \\
\hline SRW & Siqunielle Gleichstromstimulation \\
\hline UWS & Sive wakefulness syndrome \\
\hline
\end{tabular}

\section{Literatur}

1. Affolter F. Perceptual processes as prerequisites for complex human behaviour. Int Rehabil Med 1981; 3: 3-10.

2. Affolter F, Bischofberger W, Fischer L, Hofmann W, Linzmeier S, Ott-Schindele R, Peschke V, Stöhr S, Strathoff S, Trares M. Erfassung der Wirksamkeit gespürter Interaktionstherapie bei der Behandlung von Patienten mit erworbener Hirnschädigung. Neurol Rehabil 2009; 15: 12-17.

3. Andrews K, Murphy L, Munday R, Littlewood C. Misdiagnosis of the vegetative state: retrospective study in a rehabilitation unit. BMJ 1996; 313: 13-16.

4. Bagnato S, Boccagni C, Sant'Angelo A, Fingelkurts AA, Fingelkurts AA, Galardi G. Longitudinal assessment of clinical signs of recovery in patients with unresponsive wakefulness syndrome after traumatic or nontraumatic brain injury. J Neurotrauma 2016; May, ahead of print. doi:10.1089/neu.2016.4418

5. Barrons RW. Drug-induced neuromuscular blockade and myasthenia gravis. Pharmacotherapy 1997; 17: 1220-1232.

6. Bender A, Jox RJ, Grill E, Straube A, Lule D. Persistent vegetative state and minimally conscious state: a systematic review and meta-analysis of diagnostic procedures. Dtsch Arztebl Int 2015; 112: 235-242.

7. Binzer I, Schmidt HU, Timmermann T, Jochheim M, Bender A. Immediate responses to individual dialogic music therapy in patients in low awareness states. Brain Inj 2016: 1-7.

8. Bobath B. Adult Hemiplegia Evaluation and Treatment. London: Butterworth-Heinemann 1990.

9. Bodien YG, Carlowicz CA, Chatelle C, Giacino JT. Sensitivity and Specificity of the Coma Recovery Scale-Revised Total 
Score in Detection of Conscious Awareness. Arch Phys Med Rehabil 2016; 97: 490-492 e491.

10. Bruno MA, Bernheim JL, Ledoux D, Pellas F, Demertzi A, Laureys S. A survey on self-assessed well-being in a cohort of chronic locked-in syndrome patients: happy majority, miserable minority. BMJ Open 2011; 1: eoooo39.

11. Bruno MA, Ledoux D, Lambermont B, Damas F, Schnakers C, Vanhaudenhuyse A, Gosseries O, Laureys S. Comparison of the Full Outline of UnResponsiveness and Glasgow Liege Scale/Glasgow Coma Scale in an intensive care unit population. Neurocrit Care 2011; 15: 447-453.

12. Budick T, Vogel S, Jox R. Treatment decisions for patients in the vegetative state-analysis of German court decisions and law. Nervenheilkunde 2012; 31: 231-235.

13. Bundesärztekammer. Grundsätze der Bundesärztekammer zur ärztlichen Sterbebegleitung. Deutsches Ärzteblatt 2011; 107: A877-A882.

14. Chatelle C, Thibaut A, Gosseries O, Bruno MA, Demertzi A, Bernard C, Hustinx R, Tshibanda L, Bahri MA, Laureys S. Changes in cerebral metabolism in patients with a minimally conscious state responding to zolpidem. Front Hum Neurosci 2014; 8: 917.

15. Childs NL, Mercer WN, Childs HW. Accuracy of diagnosis of persistent vegetative state. Neurology 1993; 43: 1465-1467.

16. Cortese, MD, F Riganello, F Arcuri, ME Pugliese, LF Lucca, G Dolce, WG Sannita. Coma recovery scale-r: variability in the disorder of consciousness. BMC Neurol 2015; 15: 186.

17. Di H, Nie Y, Hu X, Tong Y, Heine L, Wannez S, Huang W, Yu D, He M, Thibaut A, Schnakers C, Laureys S. Assessment of visual fixation in vegetative and minimally conscious states. BMC Neurol 2014; 14: 147.

18. Donnino MW, Andersen LW, Berg KM, Reynolds JC, Nolan JP, Morley PT, Lang E, Cocchi MN, Xanthos T, Callaway CW, Soar J, IAT Force. Temperature Management After Cardiac Arrest: An Advisory Statement by the Advanced Life Support Task Force of the International Liaison Committee on Resuscitation and the American Heart Association Emergency Cardiovascular Care Committee and the Council on Cardiopulmonary, Critical Care, Perioperative and Resuscitation. Resuscitation 2016; 98: 97-104.

19. Eifert B, Maurer-Karattup P, Schorl M. Integration of intensive care treatment and neurorehabilitation in patients with disorders of consciousness: a program description and case report. Arch Phys Med Rehabil 2013; 94: 1924-1933.

20. Estraneo A, P Moretta, V Loreto, B Lanzillo, L Santoro, L Trojano. Late recovery after traumatic, anoxic, or hemorrhagic long-lasting vegetative state. Neurology 2010; 75: 239-245.

21. Estraneo A, P Moretta, V Loreto, L Santoro, L Trojano. Clinical and neuropsychological long-term outcomes after late recovery of responsiveness: a case series. Arch Phys Med Rehabil 2014; 95: 711-716.

22. Fernandez-Espejo D, C Junque, M Bernabeu, T Roig-Rovira, P Vendrell, JM Mercader. Reductions of thalamic volume and regional shape changes in the vegetative and the minimally conscious states. J Neurotrauma 2010; 27: 1187-1193.

23. Fridman EA, BZ Krimchansky, M Bonetto, T Galperin, ER Gamzu, RC Leiguarda, R Zafonte. Continuous subcutaneous apomorphine for severe disorders of consciousness after traumatic brain injury. Brain Inj 2010; 24: 636-641.

24. Ganesh S, A Guernon, L Chalcraft, B Harton, B Smith, T Louise-Bender Pape. Medical comorbidities in disorders of consciousness patients and their association with functional outcomes. Arch Phys Med Rehabil 2013; 94: 1899-1907.

25. Geocadin RG, MM Buitrago, MT Torbey, N Chandra-Strobos, MA Williams, PW Kaplan. Neurologic prognosis and withdrawal of life support after resuscitation from cardiac arrest. Neurology 2006; 67: 105-108.

26. Giacino JT, S Ashwal, N Childs, R Cranford, B Jennett, DI Katz, JP Kelly, JH Rosenberg, J Whyte, RD Zafonte, ND Zasler. The minimally conscious state: definition and diag- nostic criteria. Neurology 2002; 58: 349-353.

27. Giacino JT, DI Katz, J Whyte. Neurorehabilitation in disorders of consciousness. Semin Neurol 2013; 33: 142-156.

28. Giacino JT, C Schnakers, D Rodriguez-Moreno, K Kalmar, N Schiff, J Hirsch. Behavioral assessment in patients with disorders of consciousness: gold standard or fool's gold? Prog Brain Res 2009; 177: 33-48.

29. Giacino JT, J Whyte, E Bagiella, K Kalmar, N Childs, A Khademi, B Eifert, D Long, D I Katz, S Cho, S A Yablon, M Luther, F M Hammond, A Nordenbo, P Novak, W Mercer, P Maurer-Karattup, M Sherer. Placebo-controlled trial of amantadine for severe traumatic brain injury. N Engl J Med 2012; 366: 819-826.

30. Gill-Thwaites H, R Munday. The Sensory Modality Assessment and Rehabilitation Technique (SMART): a valid and reliable assessment for vegetative state and minimally conscious state patients. Brain Inj 2004; 18: 1255-1269.

31. Gosseries O, V Charland-Verville, M Thonnard, O Bodart, S Laureys, A Demertzi. Amantadine, apomorphine and zolpidem in the treatment of disorders of consciousness. Curr Pharm Des 2014; 20: 4167-4184.

32. Green RE, B Colella, JJ Maller, M Bayley, J Glazer, DJ Mikulis. Scale and pattern of atrophy in the chronic stages of moderate-severe TBI. Front Hum Neurosci 2014; 8: 67.

33. Grill E, AM Klein, K Howell, M Arndt, L Bodrozic, J Herzog, R Jox, E Koenig, U Mansmann, F Muller, T Muller, D Nowak, M Schaupp, A Straube, A Bender. Rationale and design of the prospective German registry of outcome in patients with severe disorders of consciousness after acute brain injury. Arch Phys Med Rehabil 2013; 94: 1870-1876.

34. Guldenmund P, A Soddu, K Baquero, A Vanhaudenhuyse, MA Bruno, O Gosseries, S Laureys, F Gomez. Structural brain injury in patients with disorders of consciousness: A voxel-based morphometry study. Brain Inj 2016; 30: 343-352.

35. Hamann GF, A Bender, B Voller, R Bühler, W von Scheidt, HC Hansen. Hypoxische Enzephalopathie. Akt Neurol 2012; 39: 309-321.

36. Hansen TS, D Jakobsen. A decision-algorithm defining the rehabilitation approach: 'Facial oral tract therapy'. Disabil Rehabil 2010; 32: 1447-1460.

37. Heinz UE, JD Rollnik. Outcome and prognosis of hypoxic brain damage patients undergoing neurological early rehabilitation. BMC Res Notes 2015; 8: 243.

38. Hoffmann B, H Karbe, C Krusch, B Müller, M Pause, M Prosiegel, W Puschendorf, J Schleep, M Spranger, D Steube, A Voss. Early Rehabilitation in Neurology and Neurosurgery: a Multicenter Study to Describe Typical Impairments and Complications Define Patient Characteristics. Akt Neurol 2006; 33: 287-296.

39. Horsting MW, MD Franken, J Meulenbelt, WA van Klei, D $\mathrm{W}$ de Lange. The etiology and outcome of non-traumatic coma in critical care: a systematic review. BMC Anesthesiol 2015; $15: 65$.

40. Howell K, E Grill, AM Klein, A Straube, A Bender. Rehabilitation outcome of anoxic-ischaemic encephalopathy survivors with prolonged disorders of consciousness. Resuscitation 2013; 84: 1409-1415.

41. Jennett B, G Teasdale. Aspects of coma after severe head injury. Lancet 1977; 1: 878-881.

42. Jox RJ, JL Bernat, S Laureys, E Racine. Disorders of consciousness: responding to requests for novel diagnostic and therapeutic interventions. Lancet Neurol 2012; 11: 732-738.

43. Jox RJ, K Kuehlmeyer, AM Klein, J Herzog, M Schaupp, DA Nowak, E Koenig, F Muller, A Bender. Diagnosis and decision making for patients with disorders of consciousness: a survey among family members. Arch Phys Med Rehabil 2015; 96: 323-330.

44. Jox RJ, K Kuhlmeyer, GD Borasio. [End-of-life decisions for patients with dementia and the vegetative state]. MMW Fortschr Med 2011; 153: 31-32, 34. 
45. Jox RJ, A Schaider, G Marckmann, G D Borasio. Medical futility at the end of life: the perspectives of intensive care and palliative care clinicians. J Med Ethics 2012; 38: 540-545.

46. Kalmar K, JT Giacino. The JFK Coma Recovery Scale--Revised. Neuropsychol Rehabil 2005; 15: 454-460.

47. Kaplan PW. Assessing the outcomes in patients with nonconvulsive status epilepticus: nonconvulsive status epilepticus is underdiagnosed, potentially overtreated, and confounded by comorbidity. J Clin Neurophysiol 1999; 16: 341-352; discussion 353.

48. Katz, D I, M Polyak, D Coughlan, M Nichols, A Roche. Natural history of recovery from brain injury after prolonged disorders of consciousness: outcome of patients admitted to inpatient rehabilitation with 1-4 year follow-up. Prog Brain Res 2009; 177: 73-88.

49. Klein AM, K Howell, A Straube, T Pfefferkorn, A Bender. Rehabilitation outcome of patients with severe and prolonged disorders of consciousness after aneurysmal subarachnoid hemorrhage (aSAH). Clin Neurol Neurosurg 2013; 115: 2136-2141.

50. Klein AM, K Howell, J Vogler, E Grill, A Straube, A Bender. Rehabilitation outcome of unconscious traumatic brain injury patients. J Neurotrauma 2013; 30: 1476-1483.

51. Klingshirn H, E Grill, A Bender, R Strobl, R Mittrach, K Braitmayer, M Muller. Quality of evidence of rehabilitation interventions in long-term care for people with severe disorders of consciousness after brain injury: A systematic review. J Rehabil Med 2015; 47: 577-585.

52. Konradi J, A Lerch, M Cataldo, T Kerz. Direct effects of Facio-Oral Tract Therapy((R)) on swallowing frequency of non-tracheotomised patients with acute neurogenic dysphagia. SAGE Open Med 2015; 3: 2050312115578958.

53. Krewer C, M Luther, E Koenig, F Muller. Tilt Table Therapies for Patients with Severe Disorders of Consciousness: A Randomized, Controlled Trial. PLoS One 2015; 10: e014318o.

54. Kuchta J, N Klug, RI Ernestus. Nonconvulsive status epilepticus as a possible cause of coma in neurosurgical intensive care. Cent Eur Neurosurg 2009; 70: 176-179.

55. Kuehlmeyer K, E Racine, N Palmour, E Hoster, G D Borasio, RJ Jox. Diagnostic and ethical challenges in disorders of consciousness and locked-in syndrome: a survey of German neurologists. J Neurol 2012; 259: 2076-2089.

56. Lancioni GE, A Bosco, MO Belardinelli, NN Singh, MF O'Reilly, J Sigafoos. An overview of intervention options for promoting adaptive behavior of persons with acquired brain injury and minimally conscious state. Res Dev Disabil 2010; 31: 1121-1134.

57. Laureys S, GG Celesia, F Cohadon, J Lavrijsen, J Leon-Carrion, WG Sannita, L Sazbon, E Schmutzhard, KR von Wild, A Zeman, G Dolce. Unresponsive wakefulness syndrome: a new name for the vegetative state or apallic syndrome. BMC Med 2010; 8: 68.

58. Leithner C, C Storm, D Hasper, CJ Ploner. Prognose der Hirnfunktion nach kardiopulmonaler Reanimation und therapeutischer Hypothermie. Akt Neurol 2012; 39: 145-154.

59. Lopez-Rolon A, A Bender, HIG Project. Hypoxia and Outcome Prediction in Early-Stage Coma (Project HOPE): an observational prospective cohort study. BMC Neurol 2015; 15: 82.

6o. Lovstad M, KF Froslie, JT Giacino, T Skandsen, A Anke, AK Schanke. Reliability and diagnostic characteristics of the JFK coma recovery scale-revised: exploring the influence of rater's level of experience. J Head Trauma Rehabil 2010; 25: 349-356.

61. Luaute J, D Maucort-Boulch, L Tell, F Quelard, T Sarraf, J Iwaz, D Boisson, C Fischer. Long-term outcomes of chronic minimally conscious and vegetative states. Neurology 2010; 75: 246-252.

62. Lule D, Q Noirhomme, SC Kleih, C Chatelle, S Halder, A Demertzi, MA Bruno, O Gosseries, A Vanhaudenhuyse, C
Schnakers, M Thonnard, A Soddu, A Kubler, S Laureys. Probing command following in patients with disorders of consciousness using a brain-computer interface. Clin Neurophysiol 2013; 124: 101-106.

63. Lule D, C Zickler, S Hacker, MA Bruno, A Demertzi, F Pellas, S Laureys, A Kubler. Life can be worth living in locked-in syndrome. Prog Brain Res 2009; 177: 339-351.

64. Magee WL, J O'Kelly. Music therapy with disorders of consciousness: current evidence and emergent evidence-based practice. Ann NY Acad Sci 2015; 1337: 256-262.

65. Maurer-Karattup P, J Giacino, M Luther, B Eifert. Diagnostik von Bewusstseinsstörungen anhand der deutschsprachigen Coma Recovery Scale-Revised (CRS-R). Neurol Rehabil 2010; 16: 232-246.

66. Monti MM, A Vanhaudenhuyse, MR Coleman, M Boly, JD Pickard, L Tshibanda, AM Owen, S Laureys. Willful modulation of brain activity in disorders of consciousness. The New England journal of medicine 2010; 362: 579-589.

67. Mura E, F Pistoia, M Sara, S Sacco, A Carolei, S Govoni. Pharmacological modulation of the state of awareness in patients with disorders of consciousness: an overview. Curr Pharm Des 2014; 20: 4121-4139.

68. Nakase-Richardson R, J Whyte, JT Giacino, S Pavawalla, SD Barnett, SA Yablon, M Sherer, K Kalmar, FM Hammond, B Greenwald, LJ Horn, R Seel, M McCarthy, J Tran, WC Walker. Longitudinal outcome of patients with disordered consciousness in the NIDRR TBI Model Systems Programs. J Neurotrauma 2012; 29: 59-65.

69. Nauwynck M, L Huyghens. Neurological complications in critically ill patients; septic encephalopathy, critical illness polyneuropathy. Acta Clin Belg 1998; 53: 92-97.

70. Nolan JP, J Soar, A Cariou, T Cronberg, VR Moulaert, C D Deakin, BW Bottiger, H Friberg, K Sunde, C Sandroni. European Resuscitation Council and European Society of Intensive Care Medicine Guidelines for Post-resuscitation Care 2015: Section 5 of the European Resuscitation Council Guidelines for Resuscitation 2015. Resuscitation 2015; 95: 202-222.

71. Ordu Gokkaya NK, M Dalyan Aras, O Oken, F Koseoglu. Fever during post-acute rehabilitation in patients with brain injury. J Rehabil Med 2005; 37: 123-125.

72. Ortega-Suhrkamp E, KR von Wild. Standards of neurologicneurosurgical early rehabilitation - a concept of the study group neurological-neurosurgical early rehabilitation. Acta Neurochir Suppl 2002; 79: 11-19.

73. Pape TL, JM Rosenow, M Steiner, T Parrish, A Guernon, B Harton, V Patil, DK Bhaumik, S McNamee, M Walker, K Froehlich, C Burress, C Odle, X Wang, AA Herrold, W Zhao, D Reda, T Mallinson, M Conneely, AJ Nemeth. PlaceboControlled Trial of Familiar Auditory Sensory Training for Acute Severe Traumatic Brain Injury: A Preliminary Report. Neurorehabil Neural Repair 2015; 29: 537-547.

74. Peterson A, D Cruse, L Naci, C Weijer, AM Owen. Risk, diagnostic error, and the clinical science of consciousness. Neuroimage Clin 2015; 7: 588-597.

75. Pickenbrock H, VU Ludwig, A Zapf, D Dressler. Conventional versus neutral positioning in central neurological disease: a multicenter randomized controlled trial. Dtsch Arztebl Int 2015; 112: 35-42.

76. Pistoia F, S Sacco, M Sara, M Franceschini, A Carolei. Intrathecal baclofen: effects on spasticity, pain, and consciousness in disorders of consciousness and locked-in syndrome. Curr Pain Headache Rep 2015; 19: 466.

77. Pohl M, K Berger, G Ketter, C Krusch, M Pause, W Puschendorf, M Schaupp, J Schleep, M Spranger, D Steube, K Scheidtmann, J Mehrholz. [Long-term course of patients in neurological rehabilitation Phase B. Results of the 6-year follow-up in a multicenter study]. Nervenarzt 2011; 82: 753-763.

78. Pohl M, M Bertram, C Bucka, M Hartwich, M Jobges, G Ketter, B Leineweber, M Mertl-Rotzer, DA Nowak, T Platz, 
JD Rollnik, K Scheidtmann, R Thomas, F von Rosen, CW Wallesch, H Woldag, P Peschel, J Mehrholz. [Course of rehabilitation in early neurological/neurosurgical rehabilitation. Results of a 2014 multi-center evaluation in Germany]. Nervenarzt 2016;

79. PVS, T M-S T F o. Medical aspects of the persistent vegetative state (2). The Multi-Society Task Force on PVS. N Engl J Med 1994; 330: 1572-1579.

8o. Rossetti AO, M Oddo, L Liaudet, P W Kaplan. Predictors of awakening from postanoxic status epilepticus after therapeutic hypothermia. Neurology 2009; 72: 744-749.

81. Rossetti AO, M Oddo, G Logroscino, P W Kaplan. Prognostication after cardiac arrest and hypothermia: a prospective study. Ann Neurol 2010; 67: 301-307.

82. Schnakers C, H Bessou, I Rubi-Fessen, A Hartmann, GR Fink, I Meister, JT Giacino, S Laureys, S Majerus. Impact of aphasia on consciousness assessment: a cross-sectional study. Neurorehabil Neural Repair 2015; 29: 41-47.

83. Schnakers C, J Giacino, K Kalmar, S Piret, E Lopez, M Boly, R Malone, S Laureys. Does the FOUR score correctly diagnose the vegetative and minimally conscious states? Ann Neurol 2006; 60: 744-745; author reply 745 .

84. Schnakers C, WL Magee, B Harris. Sensory Stimulation and Music Therapy Programs for Treating Disorders of Consciousness. Front Psychol 2016; 7: 297.

85. Schnakers C, A Vanhaudenhuyse, J Giacino, M Ventura, M Boly, S Majerus, G Moonen, S Laureys. Diagnostic accuracy of the vegetative and minimally conscious state: clinical consensus versus standardized neurobehavioral assessment. BMC Neurol 2009; 9: 35.

86. Schorl M, SJ Valerius-Kukula, TP Kemmer. Median-evoked somatosensory potentials in severe brain injury: does initial loss of cortical potentials exclude recovery? Clin Neurol Neurosurg 2014; 123: 25-33.

87. Schorr B, W Schlee, M Arndt, A Bender. Coherence in resting-state EEG as a predictor for the recovery from unresponsive wakefulness syndrome. J Neurol 2016; 263: 937-953.

88. Schorr B, W Schlee, M Arndt, D Lule, IT Kolassa, A Lopez Rolon, A Bender. Stability of auditory event-related potentials in coma research. J Neurol 2015; 262: 307-315.

89. Seel RT, J Douglas, AC Dennison, S Heaner, K Farris, C Rogers. Specialized early treatment for persons with disorders of consciousness: program components and outcomes. Arch Phys Med Rehabil 2013; 94: 1908-1923.

90. Seidl RO, R Nusser-Muller-Busch, W Hollweg, M Westhofen, A Ernst. Pilot study of a neurophysiological dysphagia therapy for neurological patients. Clin Rehabil 2007; 21: 686-697.

91. Skolnick BE, Maas AI, Narayan RK, van der Hoop RG, MacAllister T, Ward JD, Nelson NR, Stocchetti N, S T Investigators. A clinical trial of progesterone for severe traumatic brain injury. N Engl J Med 2014; 371: 2467-2476.

92. Stender J, O Gosseries, MA Bruno, V Charland-Verville, A Vanhaudenhuyse, A Demertzi, C Chatelle, M Thonnard, A Thibaut, L Heine, A Soddu, M Boly, C Schnakers, A Gjedde, $S$ Laureys. Diagnostic precision of PET imaging and functional MRI in disorders of consciousness: a clinical validation study. Lancet 2014; 384(9942): 514-522.

93. Steppacher I, S Eickhoff, T Jordanov, M Kaps, W Witzke, J Kissler. N400 predicts recovery from disorders of consciousness. Ann Neurol 2013; 73: 594-602.

94. Teasdale G, B Jennett. Assessment of coma and impaired consciousness. A practical scale. Lancet 1974; 2: 81-84.

95. Thibaut A, MA Bruno, D Ledoux, A Demertzi, S Laureys. tDCS in patients with disorders of consciousness: shamcontrolled randomized double-blind study. Neurology 2014; 82: 1112-1118.

96. Thibaut FA, C Chatelle, S Wannez, T Deltombe, J Stender, C Schnakers, S Laureys, O Gosseries. Spasticity in disorders of consciousness: a behavioral study. Eur J Phys Rehabil Med 2015; 51: 389-397.
97. Thonnard M, O Gosseries, A Demertzi, Z Lugo, A Vanhaudenhuyse, B Marie-Aurelie, C Chatelle, A Thibaut, V Charland-Verville, D Habbal, C Schnakers, S Laureys. Effect of zolpidem in chronic disorders of consciousness: a prospective open-label study. Funct Neurol 2013; 28: 259-264.

98. Tolle P, M Reimer. Do we need stimulation programs as a part of nursing care for patients in "persistent vegetative state”? A conceptual analysis. Axone 2003; 25: 20-26.

99. Tong DM, YT Zhou, GS Wang, XD Chen, TH Yang. Early prediction and outcome of septic encephalopathy in acute stroke patients with nosocomial coma. J Clin Med Res 2015; 7: 534-539.

10o. Trivedi MA, MA Ward, TM Hess, SD Gale, RJ Dempsey, HA Rowley, SC Johnson. Longitudinal changes in global brain volume between 79 and 409 days after traumatic brain injury: relationship with duration of coma. J Neurotrauma 2007; 24: 766-771.

101. van Erp WS, JC Lavrijsen, PE Vos, H Bor, S Laureys, RT Koopmans. The vegetative state: prevalence, misdiagnosis, and treatment limitations. J Am Med Dir Assoc 2015; 16: 85 e89-85 e14.

102. Vanhaudenhuyse A, C Schnakers, S Bredart, S Laureys. Assessment of visual pursuit in post-comatose states: use a mirror. J Neurol Neurosurg Psychiatry 2008; 79: 223.

103. Vincent SR. The ascending reticular activating system-from aminergic neurons to nitric oxide. J Chem Neuroanat 2000; 18: 23-30.

104. Vogler J, AM Klein, A Bender. Long-term health-related quality-of-life in patients with acquired brain injury and their caregivers. Brain Inj 2014; 28: 1381-1388.

105. Whyte J, R Myers. Incidence of clinically significant responses to zolpidem among patients with disorders of consciousness: a preliminary placebo controlled trial. American journal of physical medicine \& rehabilitation / Association of Academic Physiatrists 2009; 88: 410-418.

106. Whyte J, AM Nordenbo, K Kalmar, B Merges, E Bagiella, H Chang, S Yablon, S Cho, F Hammond, A Khademi, J Giacino. Medical complications during inpatient rehabilitation among patients with traumatic disorders of consciousness. Arch Phys Med Rehabil 2013; 94: 1877-1883.

107. Wijdicks EF. Clinical scales for comatose patients: the Glasgow Coma Scale in historical context and the new FOUR Score. Rev Neurol Dis 2006; 3: 109-117.

108. Wijdicks EF. The bare essentials: coma. Pract Neurol 2010; 10: $51-60$.

109. Wijdicks EF, WR Bamlet, BV Maramattom, EM Manno, RL McClelland. Validation of a new coma scale: The FOUR score. Ann Neurol 2005; 58: 585-593.

110. Young GB. Coma. Ann N Y Acad Sci 2009; 1157: 32-47.

\section{Interessenvermerk}

Der Autor ist Chefarzt einer neurologischen Fachklinik, die entsprechende Patienten betreut, und hat projektbezogene Fördermittel der ZNS - Hannelore Kohl Stiftung und der Else Kröner-Fresenius-Stiftung erhalten sowie der Firmen Hocoma und Hasomed.

\section{Korrespondenzadresse:}

Prof. Dr. med. Andreas Bender

Therapiezentrum Burgau

Kapuzinerstr. 34

89331 Burgau

a.bender@therapiezentrum-burgau.de 